\title{
Automated Optical Inspection System for O-Ring Based on Photometric Stereo and Machine Vision
}

\author{
Fu-Sheng Yang ${ }^{1,2}$, Chao-Ching Ho ${ }^{1, * \mathbb{D}}$ and Liang-Chia Chen ${ }^{2}$ (D) \\ 1 Graduate Institute of Manufacturing Technology, Department of Mechanical Engineering, National Taipei \\ University of Technology, 1, Sec. 3, Zhongxiao E. Rd., Taipei 10608, Taiwan; t107568008@ntut.org.tw \\ 2 Department of Mechanical Engineering, National Taiwan University, 1, Sec. 4, Roosevelt Rd., \\ Taipei 10617, Taiwan; lchen@ntu.edu.tw \\ * Correspondence: HoChao@ntut.edu.tw; Tel.: +886-2-2771-2171; Fax: +886-2-2731-7191
}

Citation: Yang, F.-S.; Ho, C.-C.; Chen, L.-C. Automated Optical Inspection System for O-Ring Based on Photometric Stereo and Machine Vision. Appl. Sci. 2021, 11, 2601. https://doi.org/10.3390/app11062601

Academic Editor: Jun-Juh Yan

Received: 5 February 2021

Accepted: 8 March 2021

Published: 15 March 2021

Publisher's Note: MDPI stays neutral with regard to jurisdictional claims in published maps and institutional affiliations.

Copyright: (c) 2021 by the authors. Licensee MDPI, Basel, Switzerland. This article is an open access article distributed under the terms and conditions of the Creative Commons Attribution (CC BY) license (https:// creativecommons.org/licenses/by/ $4.0 /)$.

\begin{abstract}
This research developed an AOI (automated optical inspection) system for the O-Ring inspection. First, an AOI system was implemented to detect surface scratches, flow marks, non-fills, and indentations on elastomer O-Rings with high surface reflection coefficients. The proposed system employed multiple light source structures to realize the photometric stereo. This method eased the identification of the O-Ring contour and Bézier control points. Then, by applying an interpolation process on these control points, we estimated the paths of the object surface. Simultaneously, regression analysis was conducted to produce a new smooth surface and then a constructed surface was compared with the model built by the photometric stereo method. The difference was deemed the candidate defect location. Overall, the detection recall rate was $100 \%$ and accuracy reached 96.56\%. This paper also developed an AOI system for the O-Ring dimension measurement. The system analyzed the contour of the O-Ring and reversely calculated the Bézier curve control points. Then, those control points were used with De Casteljau's algorithm to estimate the O-Ring dimension with high accuracy.
\end{abstract}

Keywords: photometric stereo; AOI; Bézier curve; rubber; homography; defect detection

\section{Introduction}

Recently, the automated optical inspection (AOI) system has taken the place of human inspection in highly automated manufacturing requirements. Human inspection is subjective since human inspection stability depends on operator experience and physical condition, among other factors. However, there are many reasons that make human inspection irreplaceable. For example, we cannot use a fixed light source to obtain a defect image unless we employ a light source at a particular relative direction to catch defect features on the O-Ring's surface. A such, the AOI system has experiences many ordeals in various conditions. It is difficult to exploit the traditional image process of dealing with images from cameras captured from different light sources. Therefore, it is difficult to fix a threshold for traditional image processing.

To address this problem, we studied other research that inspected rubber material. Peng et al. [1] used $7 \times 7$ kernels to find the gradient direction and convert the edge of this area to a cubic spline to measure the O-Ring dimension and to remove noise. Simultaneously, this study used the KFDA (kernel fisher discriminant analysis) to detect the O-Ring defect. Ho et al. [2] used two inspection stations to inspect components made of rubber and metal. The first inspection station provided a camera and light source to inspect defects on the top of the component. The second inspection station provided a camera and a special telecentric lens used to inspect defects on each side of the component.

The O-Ring dimension measurement was inspired by [3-6]. Works proposed by [3,4] employed an image processing method to calculate the dimension. Those papers provided a good idea to approximate contours and calculate the length of edge information. In [5], 
Dokken et al. approximated circular sections by cubic Bézier. Moreover, Lin [6] proposed a method for using the cubic Bézier curve [7] combined with a fourth-order Bézier curve. Goldapp [8] proposed a method for calculating the circular arc control point location using the Bézier curve. The approach proposed in this work is a two-stage solution in which the problem was converted from defect inspection and dimension measurement sites to a single station. The first stage was concerned with defects on the O-Ring's surface, while the second stage measured the O-Ring dimension. After repeated attempts, we find a 2.5-D defect detection system that can solve the problem we faced easier than the 2-D one. On the other hand, only a few people used defect detection by creating a 2.5D defect-free model. The defect detection method we proposed can detect any object with some trackable path Then, the algorithm will create a defect-free model. Just only by subtracting two models can be found the approximate location of a defect easily. Furthermore, the photometric stereo method gives further potential for remote monitoring [9] and machine positioning and quality checks [10].

In this paper, we propose a simple O-Ring defect detection system and dimension measurement. The proposed system is described in Section 2, and the proposed experiment flow charts are shown in Figure 1. This paper divide into two-part. One is the defect detection part, We implemented photometric stereo to reconstruct the model with sample surface (e.g., defect-free surface Figure 2a), flow marks (Figure 2b), non-fills (Figure 2c) according to ISO 3601-3:2005 [11]) and compared to optical profilometer (VR-3100, Keyence Co., Osaka, Japan) reconstruct result. Then, develop a defect detection processing and compared result. Second, we compared the algorithm we proposed to raw contours data and Douglas-Peucker algorithm. At first, we calibrate the camera and calculate the homograpy matrix with the camera parameter. Then calculate edge information with different algorithms. Finally, we compare those results with known sample dimension to calculate absolute error. The O-Rings database we collected and evaluated in Section 3. In Section 4, the experiment results and discussion with our database are shown. Furthermore, Section 5 in our conclusion.

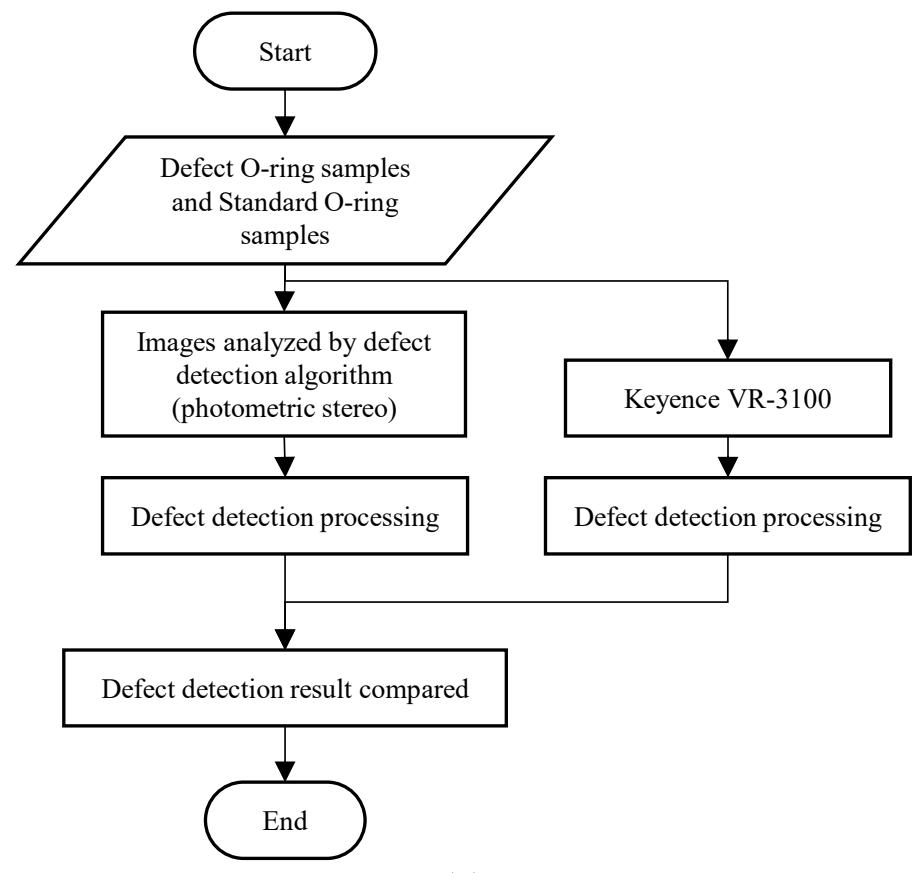

(a)

Figure 1. Cont. 


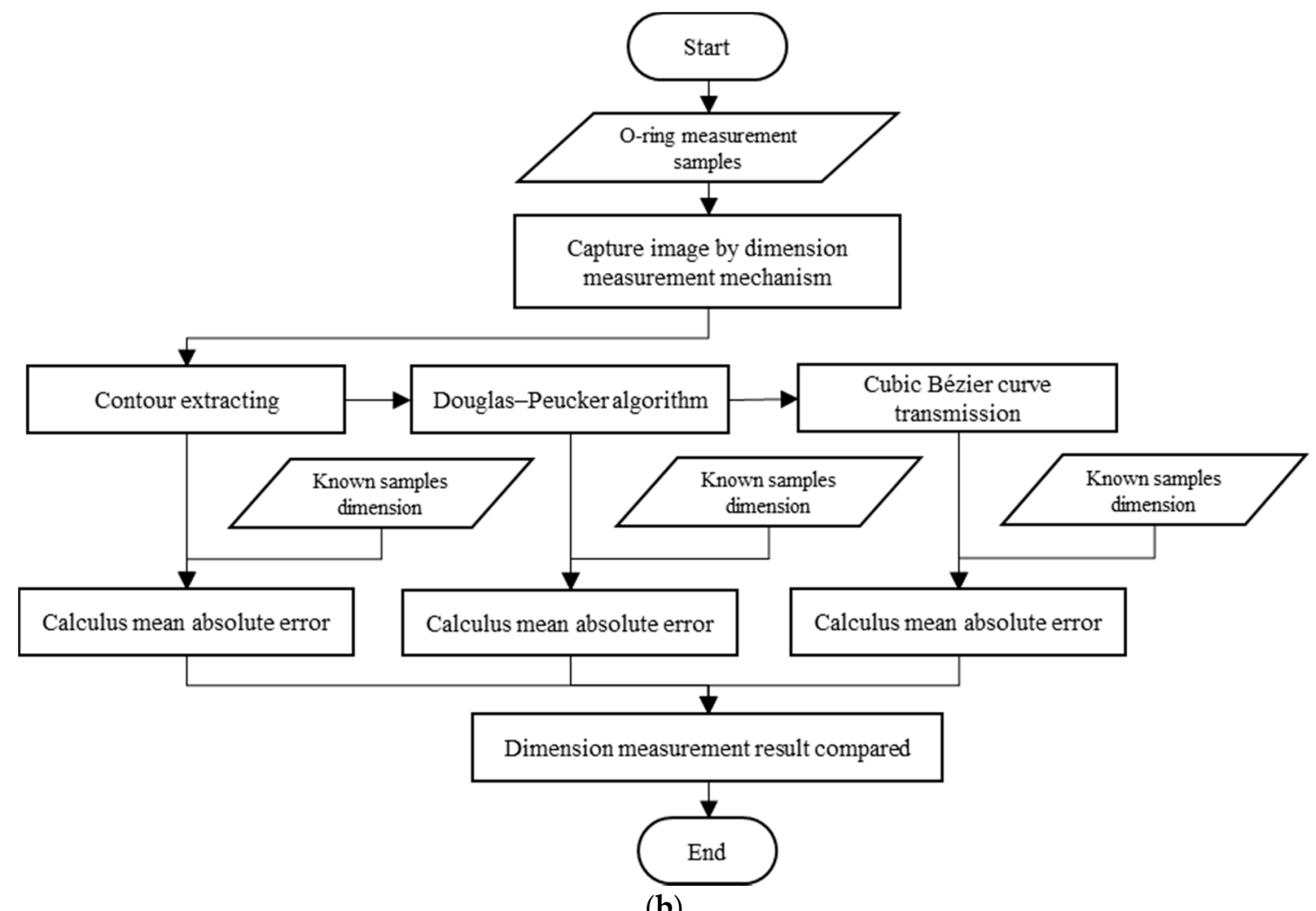

(b)

Figure 1. The proposed experiment in this paper. (a) O-Rings defect detection experiment flowchart; (b) O-Rings dimension measurement flowchart.

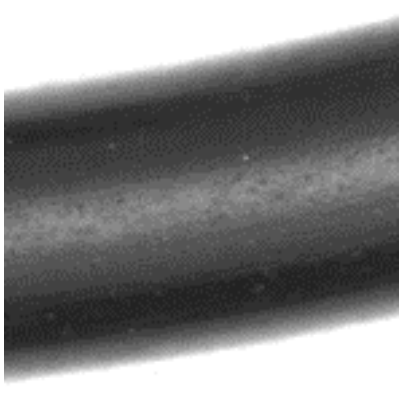

(a)

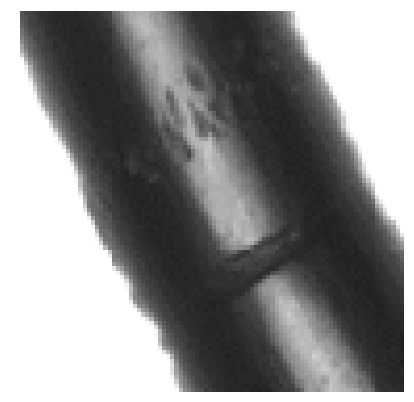

(b)

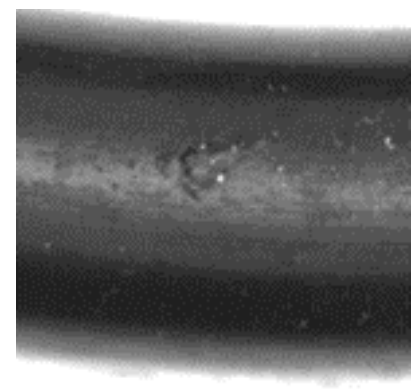

(c)

Figure 2. (a) Sample without defects; (b) sample with flow marks; (c) sample with non-fills.

\section{The Proposed System}

\subsection{Hardware Architecture}

To use the defect detection system to detect surface scratches (e.g., the defect-free surface (Figure 2a), flow marks (Figure 2b), non-fills (Figure 2c) according to ISO 36013:2005 [9]), this system provides an industrial camera and six directions of white light sources to implement a photometric stereo to meet the ISO standard (Table 1) [11]. The system provides a complementary metal-oxide semiconductor camera with Basler acA2500$16 \mathrm{um}$, which has a resolution of $2592 \times 1944$ and FOV (field of view) of $15 \times 11.25 \mathrm{~mm}^{2}$. The hardware architecture of the mechanism is shown in Figure 3a. 
Table 1. ISO 3601-3:2005 O-Ring quality acceptance criteria [9].

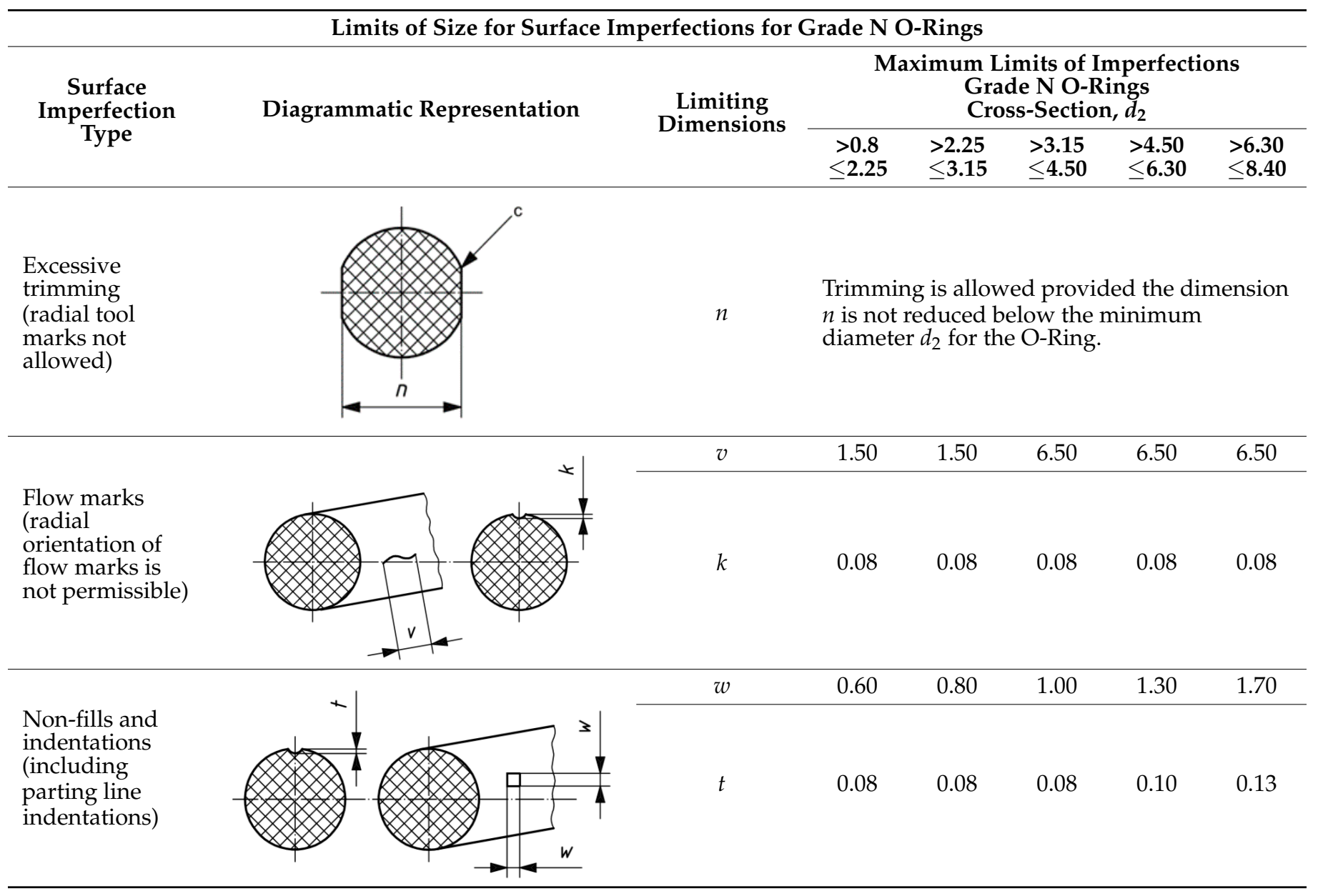
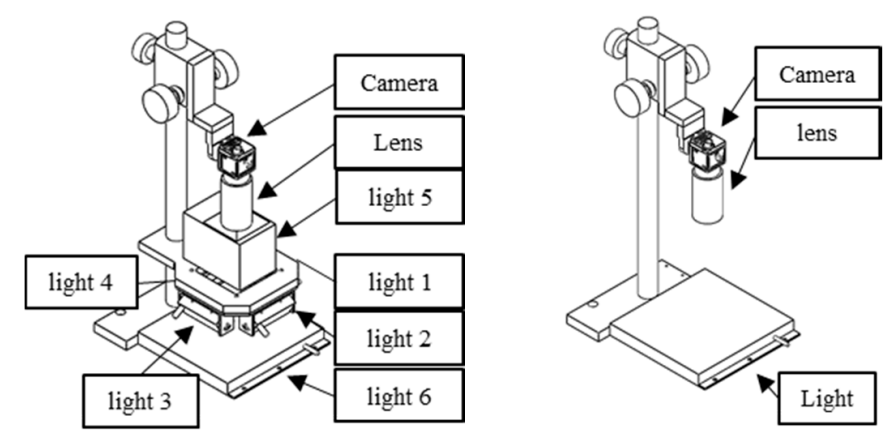

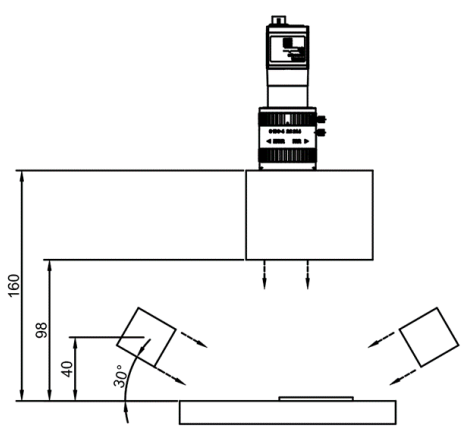

(a)

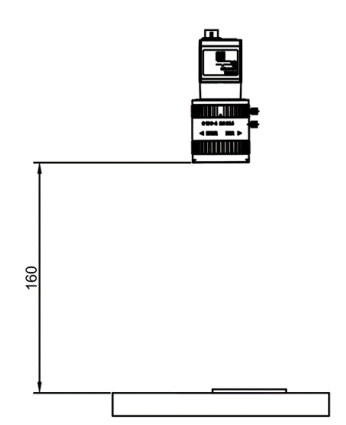

(b)

Figure 3. There are two mechanisms in this paper: (a) Defect detection system; (b) dimension measurement system. 
The dimension measurement system was used to measure the internal dimension $d_{1}$ and cross-sectional diameter $d_{2}$ of the O-Rings [12]. The $d_{1}$ and $d_{2}$ are shown in Figure 4 . The system provides a CCD camera with Basler acA2500-16 um, which had a resolution of $2592 \times 1944$ and FOV of $80 \times 60 \mathrm{~mm}$. The hardware architecture of the mechanism is shown in Figure $3 b$.
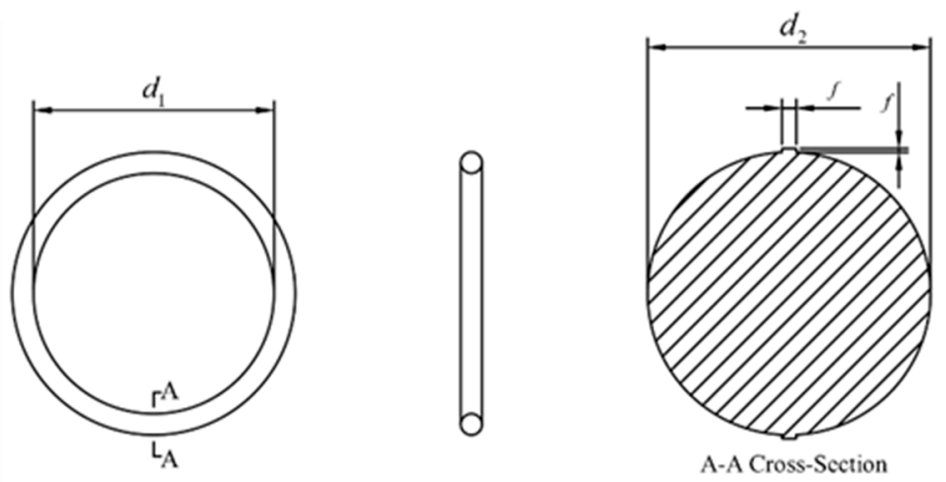

Figure 4. Schematic diagram of O-Ring dimension.

\subsection{Processing of Defect Detection}

The photometric stereo method was used to produce the 3D was obtained. Adaptive threshold using sliding windows was employed to attain the final result image $I_{R}$. The size of the sliding windows was $50 \times 50$ pixels based on the defect size. The flowchart of this study is shown in Figure 5. We used Figure 3a mechanism with a light source controller (OPT dpa2024e-8) to trigger light source and camera respectively for capture images $I_{i}$ $(i=1,2,3,4,5,6)$ (Figure 6). The light source of the mechanism came from 6 directions, from light 1 to light 6 . We employed those 6-image $\left(I_{i}, i=1,2,3,4,5,6\right)$ to implement a photometric stereo [13] as shown below:

1. Make a binary mask (Figure $7 I_{6}$ ) by using $I_{6}$. Then, we used this mask to flit background information to reduce background noise effectively.

2. Normalize image by using function (1). Which $I(x, y)$ is a grayscale pixel value at position $(x, y)$ of image.

$$
\bar{I}(x, y)_{i}=\frac{I(x, y)_{i}}{\operatorname{Max}(I(x, y))}(i=1,2,3,4,5,6)
$$

3. Set a threshold in 100 to binary image Ii $(i=1,2,3,4,5,6)$ (Figure 7) and calculate image moment $(\bar{x}, \bar{y})_{i}$ with images Ii $(i=1,2,3,4,5,6)$ (Figure 7 ) by function (2).

$$
(\bar{x}, \bar{y})=\left(\frac{M_{10}}{M_{00}}, \frac{M_{01}}{M_{00}}\right)
$$

4. Define image moment $(\bar{x}, \bar{y})_{6}$ as centroid and calculate maximum distance to mask edge $r$.

$$
r=\operatorname{Max}\left[(x, y)-(\bar{x}, \bar{y})_{6}\right] \text { when } I(x, y)>100
$$

5. Calculate light source direction vectors $\vec{L}$ using the function (8).

$$
\begin{gathered}
(d x, d y)_{i}=(\bar{x}, \bar{y})_{i}-(\bar{x}, \bar{y})_{6}(i=1,2,3,4,5,6) \\
n_{i}=\left[\frac{d x_{i}}{r},-\frac{d y_{i}}{r}, \frac{\sqrt{r^{2}-d x_{i}^{2}-d y_{i}^{2}}}{r}\right](i=1,2,3,4,5,6) \\
R=[0,0,1] \\
L_{i}=2 \times\left(n_{i} \cdot R\right) \times n_{i}-R(i=1,2,3,4,5,6)
\end{gathered}
$$




$$
\vec{L}=\left[L_{1}, L_{2}, L_{3}, L_{4}, L_{5}, L_{6}\right]
$$

6. Define normal relative surface normal image $N$ by using the function (10).

$$
\begin{gathered}
\vec{I}(x, y)=\left[\bar{I}(x, y)_{1}, \bar{I}(x, y)_{2}, \bar{I}(x, y)_{3}, \bar{I}(x, y)_{4}, \bar{I}(x, y)_{5}, \bar{I}(x, y)_{6}\right] \\
N(x, y)=\left(\vec{L}^{T} \vec{L}\right)^{-1} \vec{L}^{T} \vec{I}(x, y)
\end{gathered}
$$

7. Calculate unit vector field $\bar{N}$ (Figure 8 ) by the function (11).

$$
\bar{N}(x, y)=\frac{N(x, y)}{\|N(x, y)\|}
$$

8. First, we transfer unit vector field $\bar{N}$ to triangle mesh. Then define each vertex point as $P i(i=0,1,2)$ as function (12). Furthermore, each vertex point's relative surface normal vector $\bar{N}_{i}(i=0,1,2)$. At last, we can define $\left[N_{x}, N_{y}, N_{z}\right]$ by using the function (13).

$$
\begin{gathered}
P_{i}=\left(x_{i}, y_{i}, z_{i}\right)(i=0,1,2) \\
{\left[N_{x}, N_{y}, N_{z}\right]=\frac{\left(N_{0}+2 N_{1}+N_{2}\right)}{4}}
\end{gathered}
$$

9. Now, we can solve each vertex point's relative surface normal vector $\bar{N}_{i}(i=0,1,2)$ by using the function (14) and unit vector field $\bar{N}$.

$$
\bar{N}_{i}=\left[\begin{array}{c}
N_{x}=\left(y_{1}-y_{0}\right)\left(z_{2}-z_{0}\right)-\left(z_{1}-z_{0}\right)\left(y_{2}-y_{0}\right) \\
N_{y}=\left(z_{1}-z_{0}\right)\left(x_{2}-x_{0}\right)-\left(x_{1}-x_{0}\right)\left(y_{2}-y_{0}\right) \\
N_{z}=\left(x_{1}-x_{0}\right)\left(y_{2}-y_{0}\right)-\left(y_{1}-y_{0}\right)\left(x_{2}-x_{0}\right)
\end{array}\right]
$$

10. The deep of the triangle mesh can be rewritten as an overdetermined set. So, in the image resolution $M \times N$ would include $2(M-1)(N-1)$ the amount triangle. That is $2(M-1)(N-1)$ amount linear equation. Each triangle contains two linear equations as the function (16).

$$
\begin{gathered}
\alpha=\frac{\left[\left(x_{1}-x_{0}\right)\left(y_{2}-y_{0}\right)-\left(y_{1}-y_{0}\right)\left(x_{2}-x_{0}\right)\right]}{N_{z}} \\
\bar{N}_{i}=\left[\begin{array}{l}
\alpha N_{x}=\left(y_{1}-y_{0}\right)\left(z_{2}-z_{0}\right)-\left(z_{1}-z_{0}\right)\left(y_{2}-y_{0}\right) \\
\alpha N_{y}=\left(z_{1}-z_{0}\right)\left(x_{2}-x_{0}\right)-\left(x_{1}-x_{0}\right)\left(y_{2}-y_{0}\right)
\end{array}\right]
\end{gathered}
$$

11. We define A equal all of constant relative with $z$, and B equal $\alpha N_{x}$ and $\alpha N_{y}$. Then, we can get the relative height of each pixel $\mathrm{Z}$ by using the function (14).

$$
Z=\left(A^{T} A\right)^{-1} A^{T} B
$$

12. At last, we create a 2.5-D model by using the photometric stereo method. The result is shown in Figure 9a. With the photometric stereo method, errors resulting from surface dirt in the inspection process were effectively prevented.

On the other hand, we used the image from button light of defect detection mechanism $\left(I_{6}\right.$, Figure $\left.9 b\right)$ to produce a binary image (Figure $\left.9 c\right)$. Moreover, this binary image was one of the materials used to produce a smooth surface model $M_{\mathrm{S}}$ (Figure 9d). 


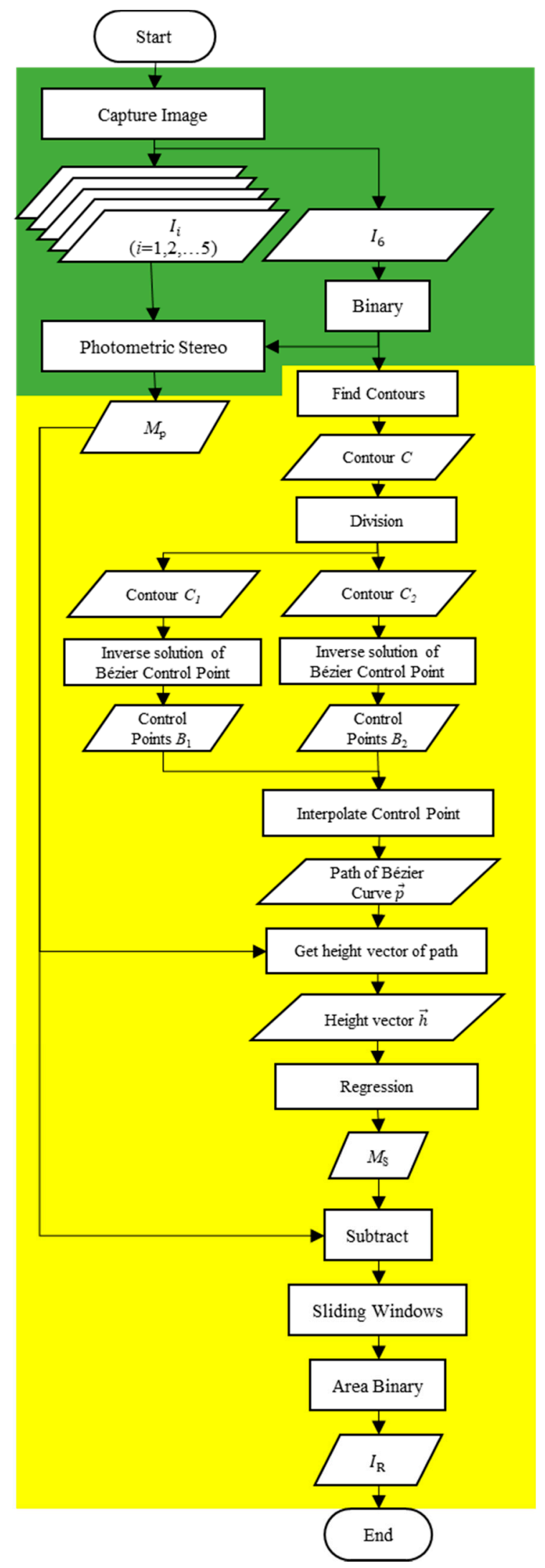

Figure 5. Flowchart of the O-Rings defect detection process. The green region is image capture relative processing and the yellow region is defect detection relative processing. Which $M_{\mathrm{P}}$ and $I_{6}$ replace with result of optical profilometer reconstruction when the verification stage. 


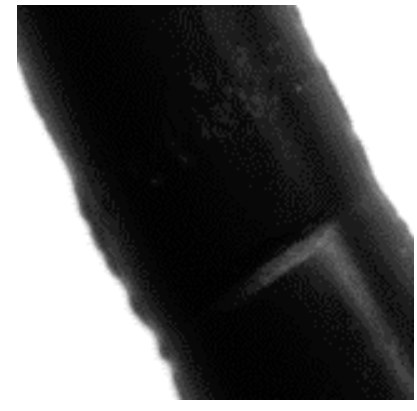

$I_{1}$

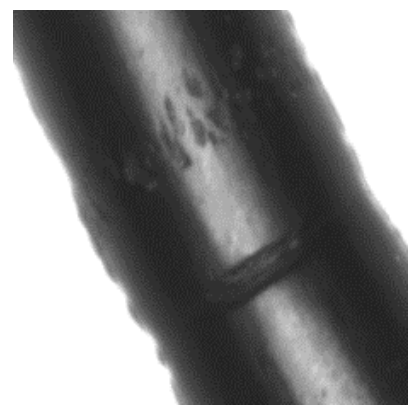

$I_{4}$

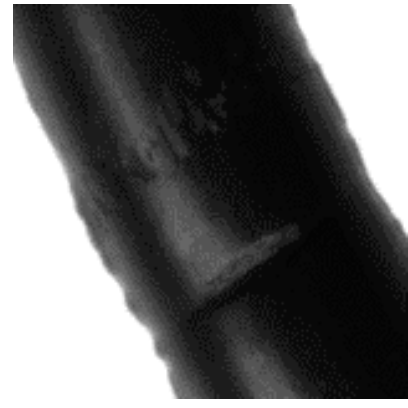

$I_{2}$

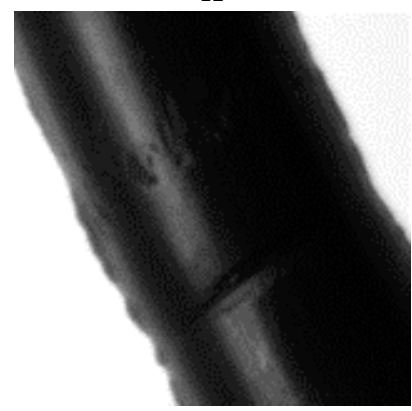

$I_{5}$

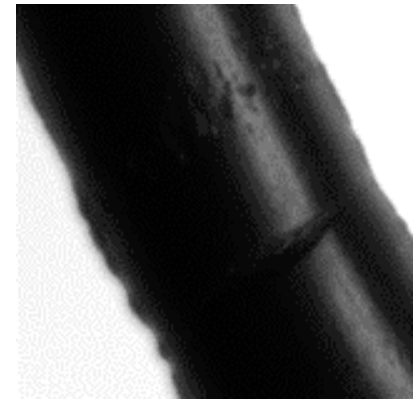

$I_{3}$

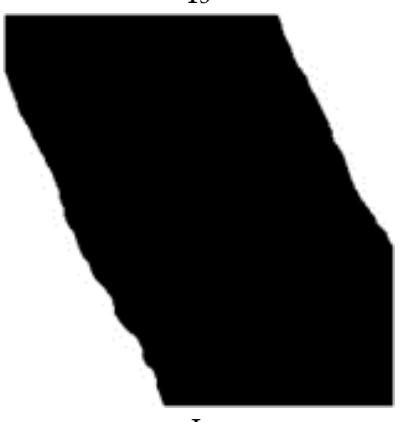

I 6

Figure 6. Images $I_{i}(i=1,2,3,4,5,6)$ from the camera of defection detection system capture.

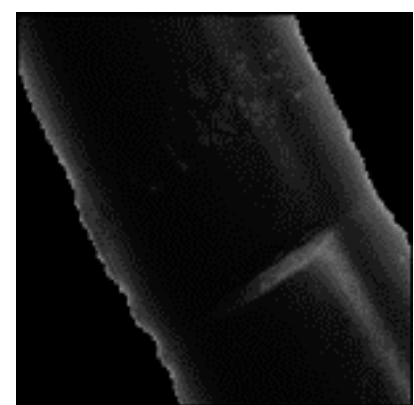

$I_{1}$

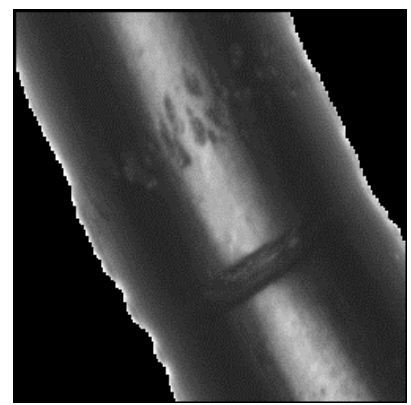

$I_{4}$

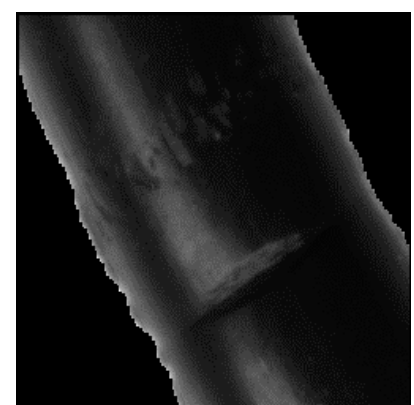

$I_{2}$

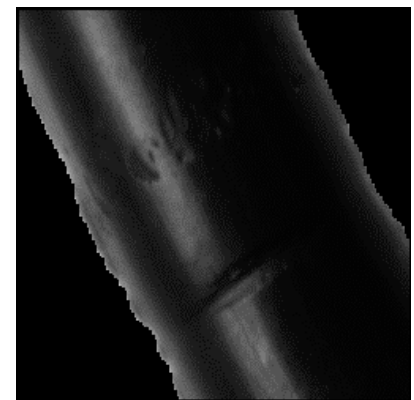

$I_{5}$

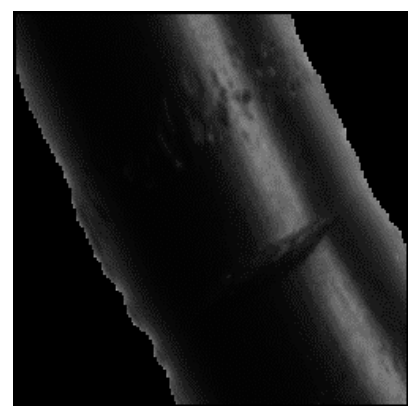

$I_{3}$

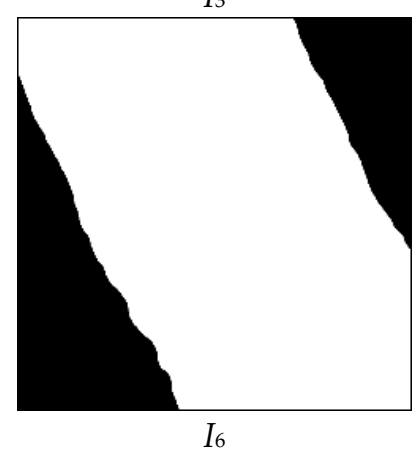

Figure 7. A set images $I_{i}(i=1,2,3,4,5,6)$ without background information. 


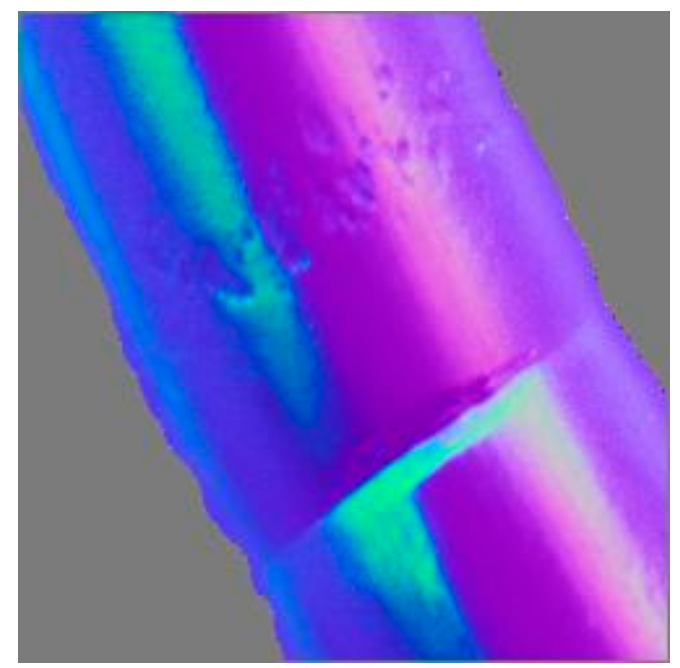

Figure 8. Unit vector field $\bar{N}$ by using function (11).

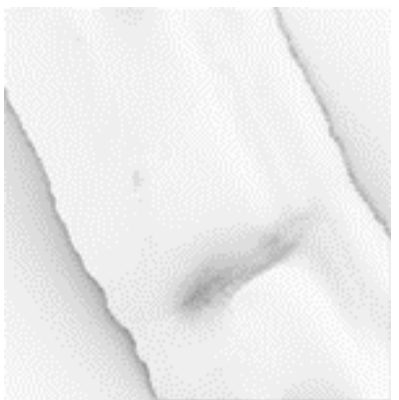

(a)

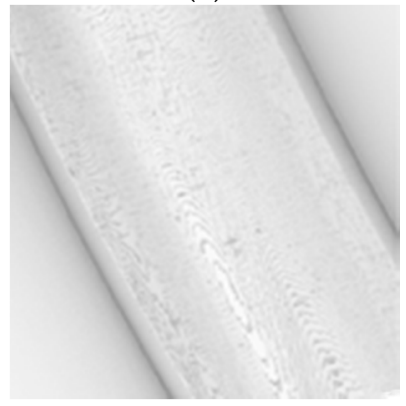

(d)

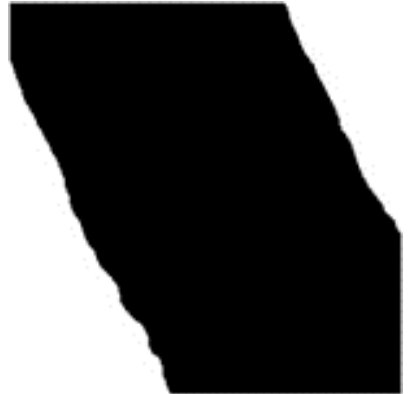

(b)

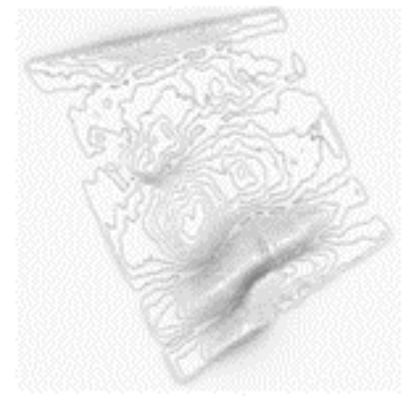

(e)

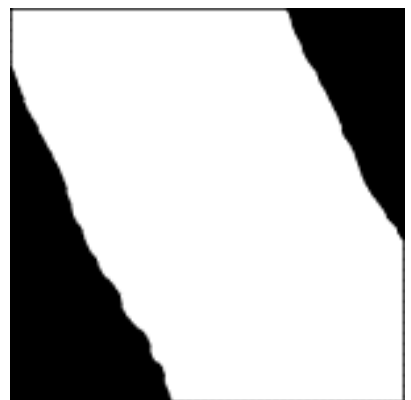

(c)

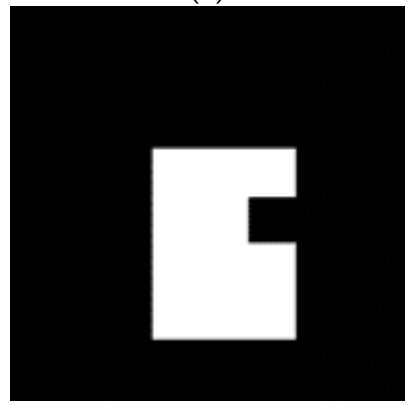

(f)

Figure 9. Each stage output of defect detection processing: (a) Model of photometric stereo produce; (b) $I_{6}$, this image capture with the button light source in defect detection mechanism (Figure 3); (c) binarization and inverse image of (b); (d) smooth model $M_{\mathrm{S}}$, created by Figure 5; (e) result of (d) subtracted from (e); (f) candidate defect area, which create by sliding windows.

We generated two factors to produce smooth surface model. One was a binary image of $I_{6}$ (Figure 9c) and the other one was the photometric stereo model $M_{\mathrm{S}}$. First, we extract contours [14] from Figure 9c. the method of find contour can be explained simply as a curve joining all the continuous points (along the boundary), having same color or intensity. The contours extracted based on chain code 8-connectivity are shown in Figure 10a. then, we used cubic Bézier curve to describe to contours. Before the transformation between contour and cubic Bézier curve. we named all of the control points of the cubic Bézier curve shown in Figure 11. Therefore, we defined the cubic Bézier curve in Equation (18), which we obtained with starting point $p_{00}$, end point $p_{03}$, tracking points $p_{30}$ with corresponding parameter $\omega$, all of $\omega$ held values that varied between 0 and 1 . Which parameter $\omega$ is used to describe cubic Bézier curve. After that, we obtained $p_{01}$ and $p_{02}$ using the solution of 
simultaneous Equation (19). We used the contour start point $p_{00}$, end point $p_{03}$, two tracking points $p_{30}$ with corresponding parameter $\omega$ to solute Equation (19). Which tracking points selected by one-third point and two-thirds points of contour points. In Figure 10b, the red points are the cubic Bézier curve's control points and the green curve is the cubic Bézier curve. In other words, we used the solution of the simultaneous Equation (19) to acquire $B_{1}$ and $B_{2}$. Moreover, we acquired the path vector $\vec{p}$ of the O-Ring by interpolating both $B_{1}$ and $B_{2}$.

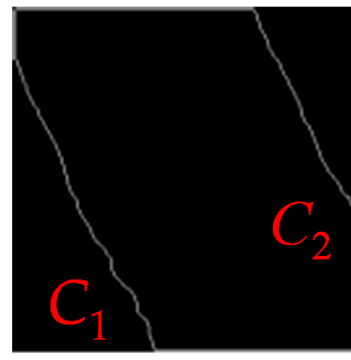

(a)

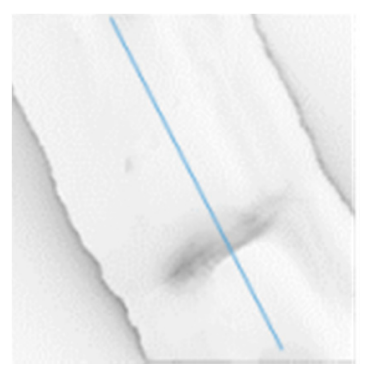

(c)

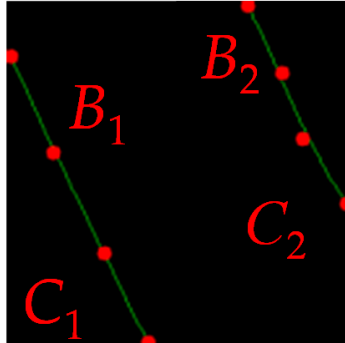

(b)

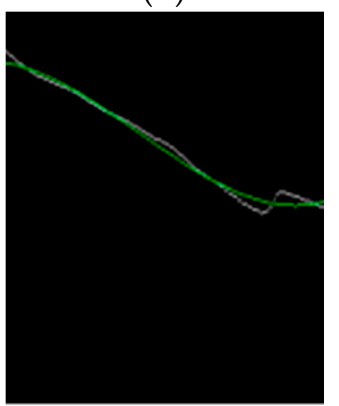

(d)

Figure 10. Each stage output of create smooth model processing: (a) Contour of $I_{6}$ binarization, external contour $C_{1}$, and internal contour $C_{2} ;(\mathbf{b})$ Bézier curve and control points $B_{1}$ converted by $C_{1}$ and Bézier curve/control points $B_{2}$ converted by $C_{2} ;$ (c) path $\vec{p}$ of interpolated between $B_{1}$ and $B_{2}$; (d) height vector $\vec{h}$ above $p_{i}$ and regression analysis result of $\vec{h}$.

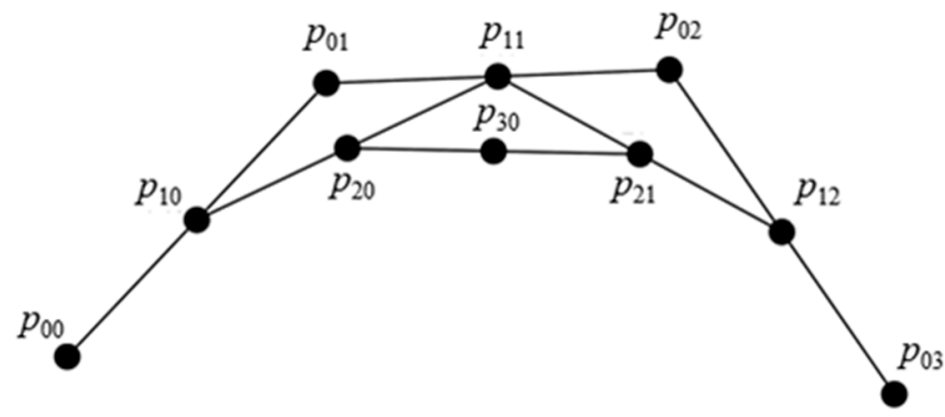

Figure 11. Control points of the cubic Bézier curve.

$$
\begin{gathered}
p_{30}=(1-\omega)^{3} p_{00}+3 \omega(1-\omega)^{2} p_{01}+3 \omega^{2}(1-\omega) p_{02}+\omega^{3} p_{03} \omega \in[0,1] \\
3 \omega(1-\omega)^{2} p_{01}+3 \omega^{2}(1-\omega) p_{02}=p_{30}-(1-\omega)^{3} p_{00}-\omega^{3} p_{03}
\end{gathered}
$$

Secondly, we obtained several vectors of height $\vec{h}$ (Figure 10c by using the path $\vec{p}$ as a factor. Thus, we calculated all $\vec{h}$ to get the result (Figure $10 \mathrm{~d}$ ) by performing a regression analysis. After that, we can acquire a smooth model of the photometric stereo $M_{\mathrm{S}}$ (Figure 9d). 
We obtained the result of $M_{\mathrm{S}}$ by subtracting from the photometric stereo model (Figure 9e). Moreover, we acquired the candidate defect area using sliding windows to eliminate model noise generated in the subtraction process. Furthermore, the candidate defect area is shown in Figure $9 \mathrm{f}$.

\subsection{Processing of Dimension Measurement}

\subsubsection{Pre-Processing of Dimension Measurement}

Camera calibration [15] was employed to calculate the homography [16] before the dimension measurement process. The pre-processing flow chat is shown in Figure 12 and the projected relation between the image plane and object plane is shown in Figure 13.

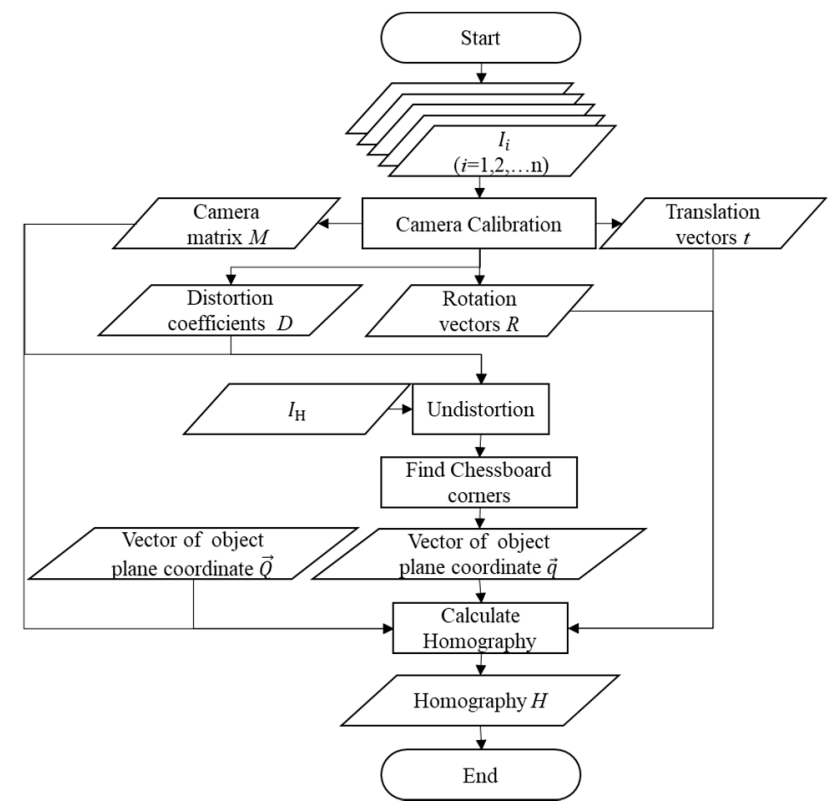

(a)

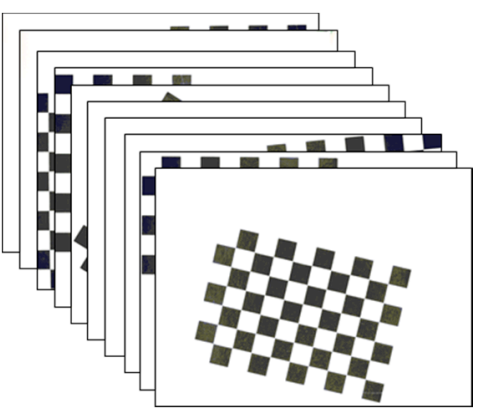

(b)

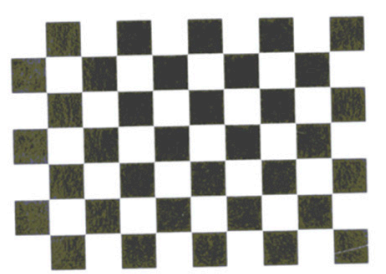

(c)

Figure 12. (a) Flowchart of pre-processing the dimension measurement; (b) images for camera calibration $I_{i} ;$ (c) image for calculating homography $I_{\mathrm{H}}$.

In the camera calibration process, we acquired the camera matrix $M$, distortion coefficients $D$, rotation vectors $R=\left(r_{1}, r_{2}, r_{3}\right)$, and translation vector $t=\left(t_{\mathrm{x}}, t_{\mathrm{y}}, t_{\mathrm{z}}\right)$ using images $I_{i}(i=1,2, \ldots, n)$ (Figure 12b) to calibrate the camera. According to the method proposed in $[15,16]$, we acquired homography $H$ by using Equation (20) and the image used to calculate homography $I_{\mathrm{H}}$ (Figure $12 \mathrm{c}$ ). $q$ is the image plane coordinates and the $Q$ is the object plane coordinate in (20). 


$$
\begin{gathered}
{\left[\begin{array}{l}
x \\
y \\
z
\end{array}\right]=s M\left[\begin{array}{llll}
r_{1} & r_{2} & r_{3} & t
\end{array}\right]\left[\begin{array}{l}
X \\
Y \\
0 \\
1
\end{array}\right] \Rightarrow s M\left[\begin{array}{lll}
r_{1} & r_{2} & t
\end{array}\right]\left[\begin{array}{l}
X \\
Y \\
1
\end{array}\right]} \\
\Rightarrow q=H Q \text { where } H=s M\left[\begin{array}{lll}
r_{1} & r_{2} & t
\end{array}\right]
\end{gathered}
$$

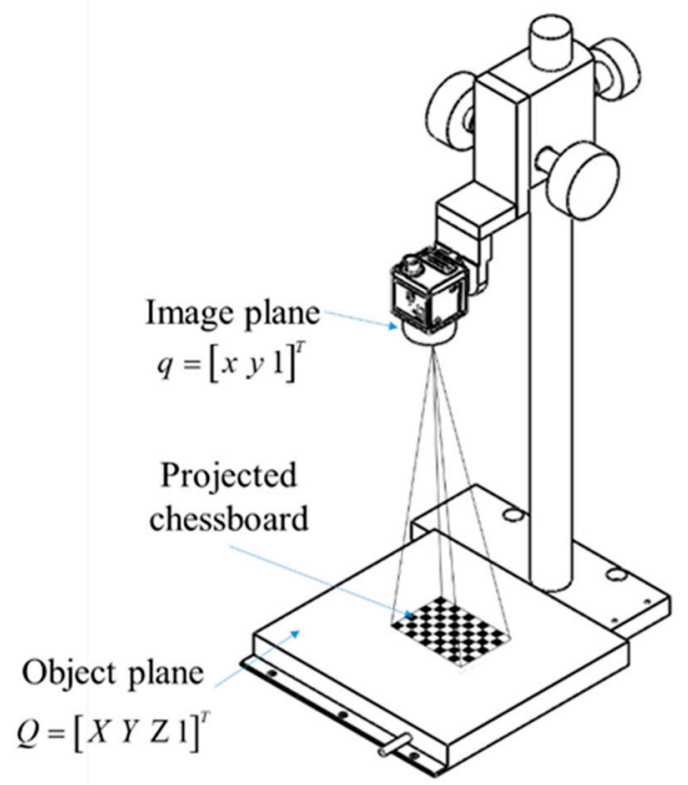

Figure 13. The schematic diagram of projected relation between the image plane and object plane in measurement mechanism (Figure 3b).

\subsubsection{Dimension Measurement Processes}

The dimension measurement process is shown in Figure 14. This process starts with image $I$, which is captured by the camera. Then, undistort $I$ (Figure 15a) is acquired using distortion coefficients $D$ and camera matrix $M$. Finally, the contours [14] (Figure 15c) are acquired using the $I$ binarization image $I_{\mathrm{b}}$ (Figure $15 \mathrm{~b}$ ).

First, we divided the contours into two parts using hierarchy [14]. One was the internal contour (Figure 15c), the other was the external contour. Then, we converted those contours into the cubic Bézier curve. In the beginning of the process, we converted the contour into the Bézier curve, and divided contour $C$ (Figure 15c) into contour $C_{i}$ (the green line between Figure 15e, red points) using approximate points $\vec{A}$ (Figure $15 \mathrm{~d}$ ) from the Douglas-Peucker algorithm [17]. The Douglas-Peucker algorithm is shown below:

1. Find the start point $P_{\mathrm{A}}$ and end point $P_{\mathrm{B}}$ of object contour $C$ (Figure 16a);

2. Connect start point $P_{\mathrm{A}}$ and end point $P_{\mathrm{B}}$. Then, find the furthest point $P_{\mathrm{C}}$ and calculate the distance $D$ from the point $P_{C}$ to the line $\overline{P_{A} P_{B}}$ (Figure 16b).

3. If the distance $D$ from the point $P_{C}$ to the line $\overline{P_{A} P_{B}}$ is bigger than the threshold we settled, divide the contour into two parts. Then, calculate the distance $D$ from the point to the line, respectively (Figure 16c).

4. Else if all of the distance $D$ from the point to the line is smaller than the threshold finish the approximate operation. 


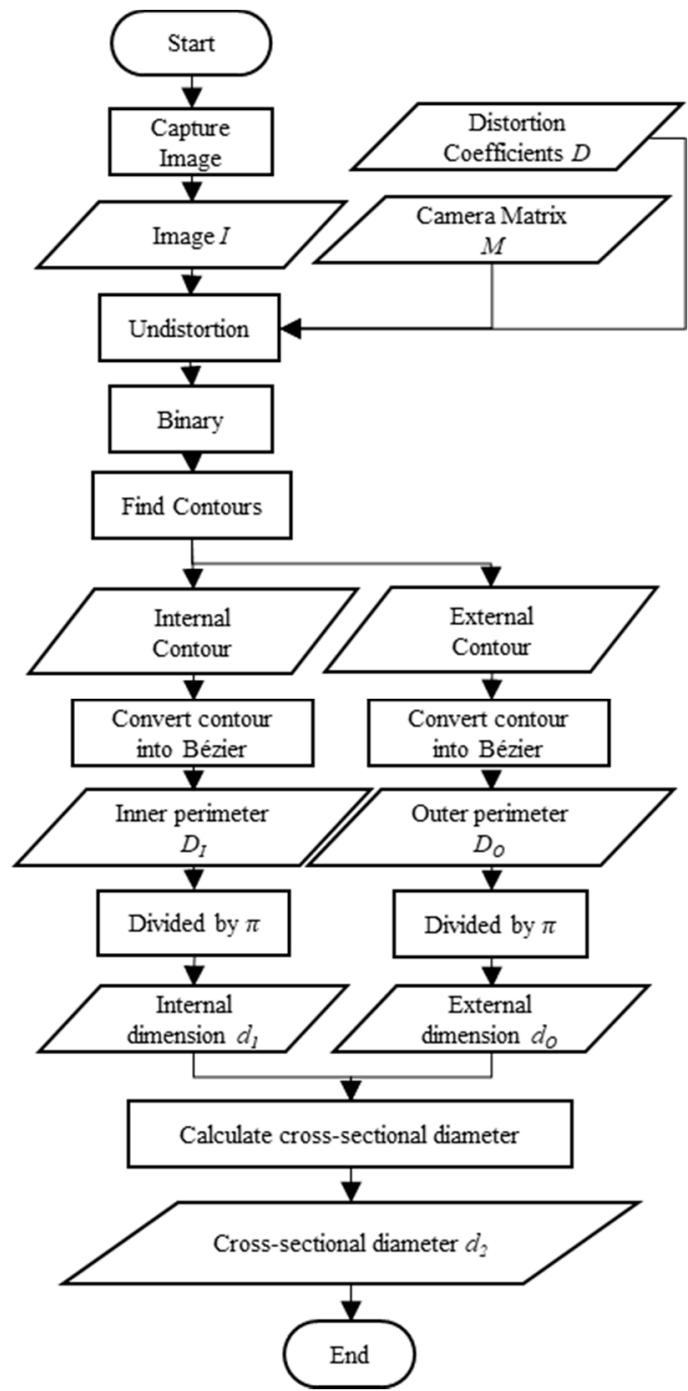

Figure 14. Flowchart of the dimension measurement.

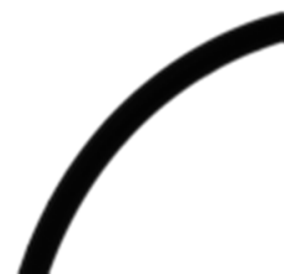

(a)

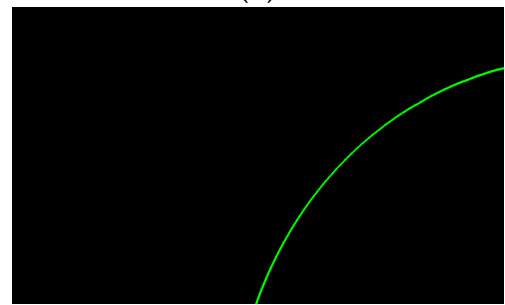

(c)

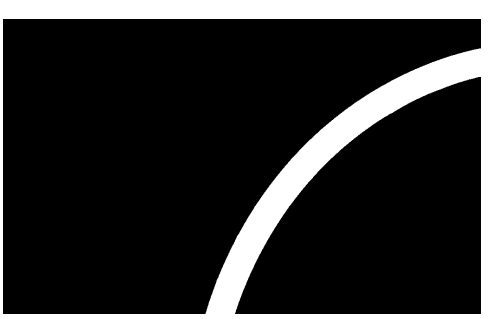

(b)

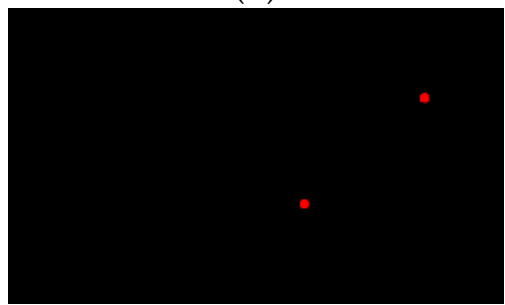

(d)

Figure 15. Cont. 


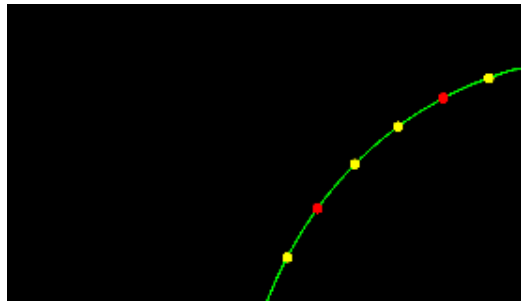

(e)

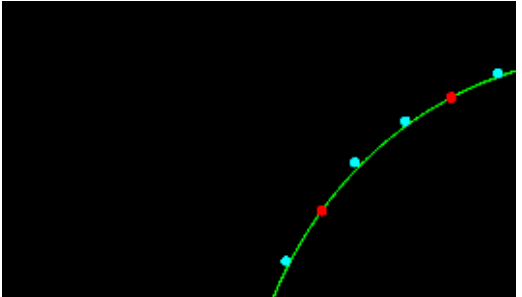

(f)

Figure 15. Each stage output of dimension measurement processing: (a) The image $I$ from camera capture; (b) the binarization and inverse image $I_{b}$; (c) internal contour of the O-Ring; (d) approximate points $\vec{A}$; (e) schematic diagram of the divided contour; (f) result of the converted cubic Bézier control points.

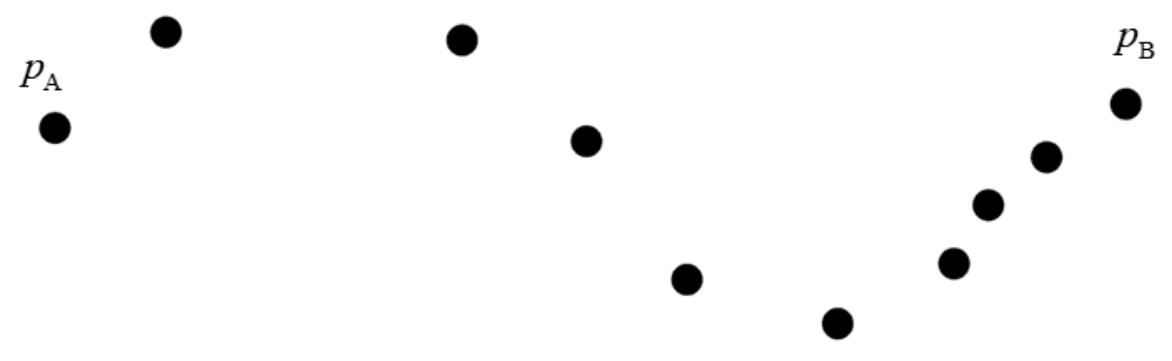

(a)

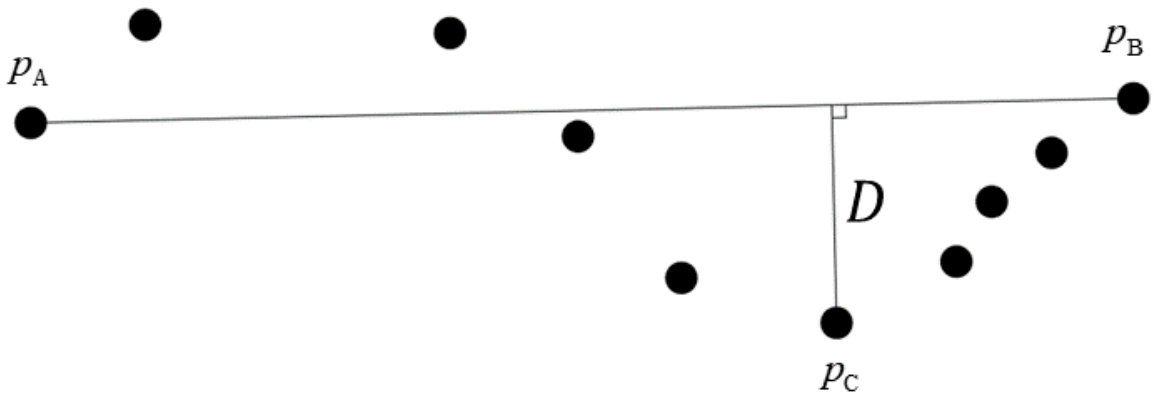

(b)

Figure 16. Cont. 


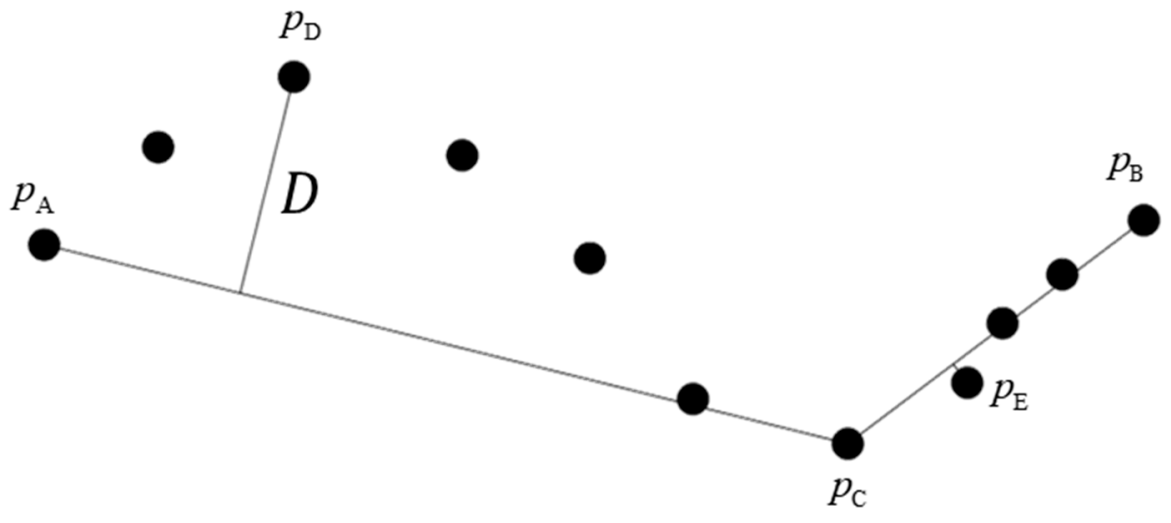

(c)

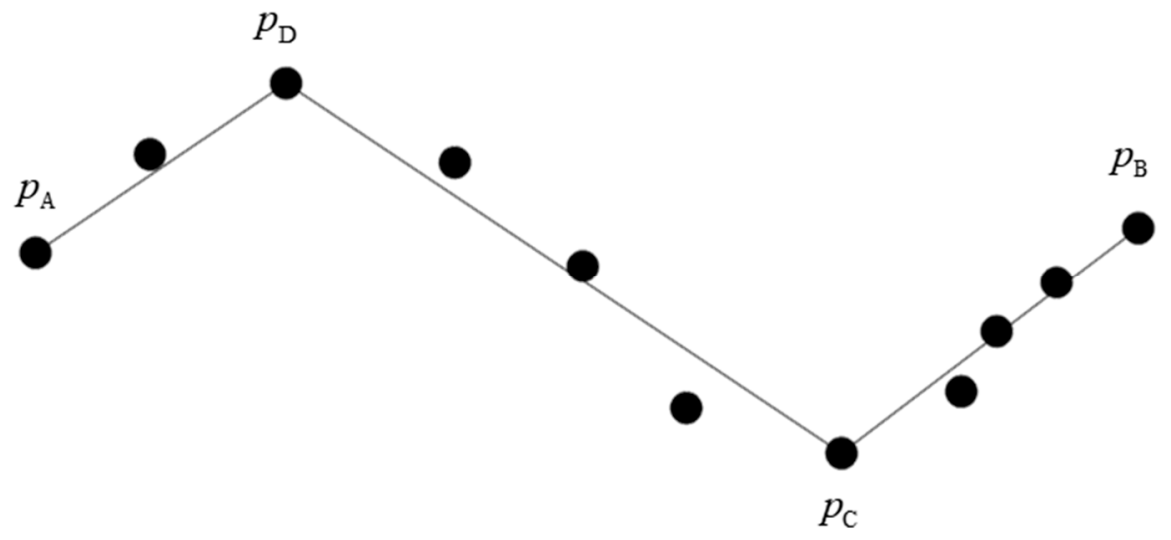

(d)

Figure 16. Schematic diagram of Douglas-Peucker algorithm: (a) A set of contour points and start point $P_{\mathrm{A}}$ and end point $P_{\mathrm{B}} ;(\mathbf{b})$ Connect start point $P_{\mathrm{A}}$ and end point $P_{\mathrm{B}}$. Then, find the furthest point $P_{C}$ and calculate the distance $D$ from the point $P_{C}$ to the line $\overline{P_{A} P_{B}} ;$ (c) If the distance $D$ from the point $P_{C}$ to the line $\overline{P_{A} P_{B}}$ is bigger than the threshold we settled, divide the contour into two parts. Then, calculate the distance $D$ from the point to the line, respectively; (d) Else if all of the distance $D$ from the point to the line is smaller than the threshold finish the approximate operation.

By using the Douglas-Peucker algorithm, we could ignore the $I_{\mathrm{b}}$ (Figure 15b) edge noise and O-Ring burrs to obtain a perimeter. Though the perimeter was more accurate than the calculated contour, it cost us too much information regarding the real edge. As a result, we proposed a more accurate solution using the cubic Bézier curve to approximate each $C_{i}$. We employed Equation (19) to acquire the cubic Bézier control points (Figure 15f) with each $C_{i}$ and projected control points using homography $H$ [16]. Finally, the arc length was calculated using the Euclidean distance via de Casteljau's algorithm [18]. The process of the cubic Bézier curve conversion is shown in Figure 17. 


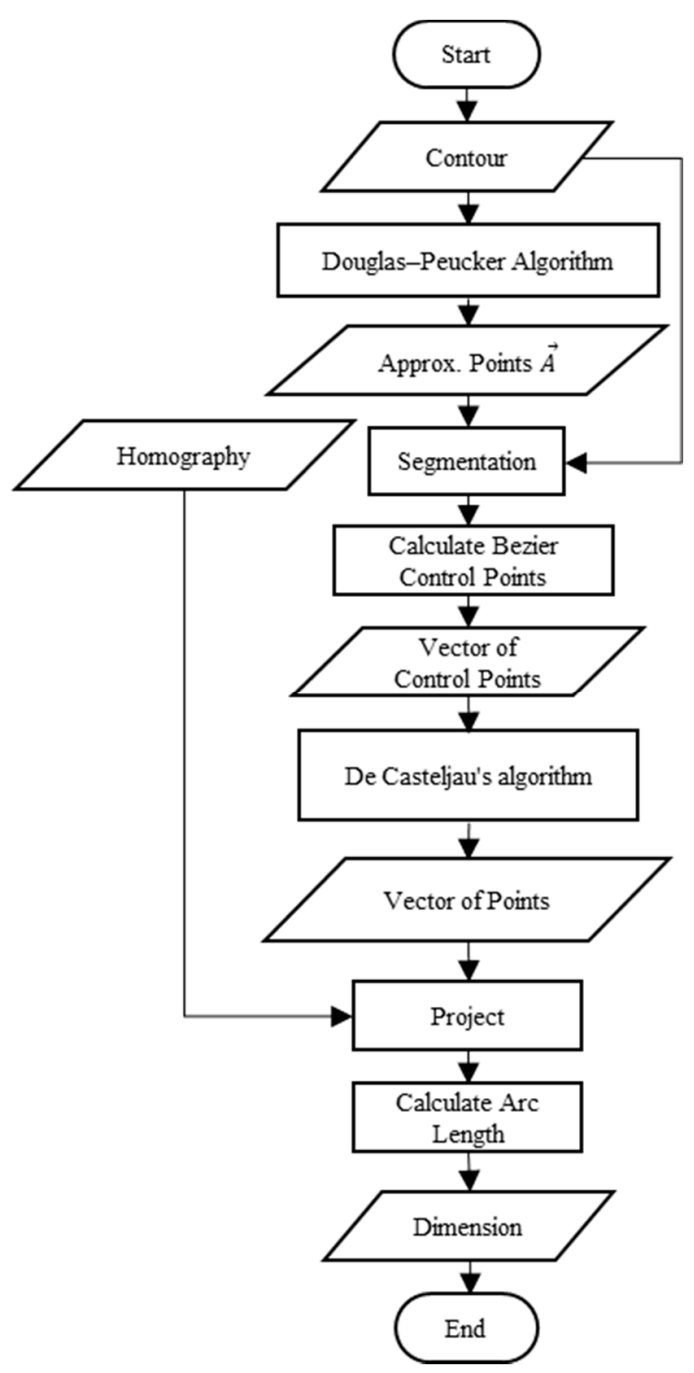

Figure 17. Flowchart of the contour converted the Bézier curve.

\section{O-Ring Database}

To verify the proposed defect detection method and dimension measure measurement, and we established five databases of O-Rings. Two databases for defect detection and three for dimension measurements were available.

\subsection{Database for Defect Detection}

\subsubsection{DPS (Database of Photometric Stereo Model)}

There were 160 defects and 160 non-defects included. The defect samples included 60 samples with flow marks and 100 samples with non-fills and indentations. Each sample had six images $I_{i}(i=1,2,3,4,5,6)$ to implement the photometric stereo and verify the defect detection process. A sample of this database is shown in Figure 6. There are several different dimensions and cross-section dimension O-Ring samples. The schematic diagram of those samples is shown in Figure 18.

We put O-Rings above the button light source in the defect detection mechanism (Figure 3a). Furthermore, collecting sample images capture by trigger each light source and camera in order. Each sample was collected in the same light parameter, camera parameter. Then, we execute the defect detection we proposed in the same condition with every sample.

In this experiment, we use a light source controller (OPT dpa2024e-8) to modify light intensity in the defect detection system (Figure 2a). Each channel of the light source set on 80. The camera exposure time set on 3000 us. By subtracting the photometric stereo model $M_{\mathrm{P}}$ and smooth surface model $M_{\mathrm{S}}$. The deviation model $M_{\mathrm{D}}$ was produced. Then we used 
the $50 \times 50$ pixels sliding windows and set the threshold on 80 in 8-bit matrix data for the deviation model $M_{\mathrm{D}}$ to create defect candidate area. If there are five candidate areas were connected. Then, label the sample as a defect. The threshold and the camera parameter by experiment obtain. Because the photometric stereo method in this paper was based on regression, Which type of method can't provide accurate relative height, the detection processing is shown in Figure 19.
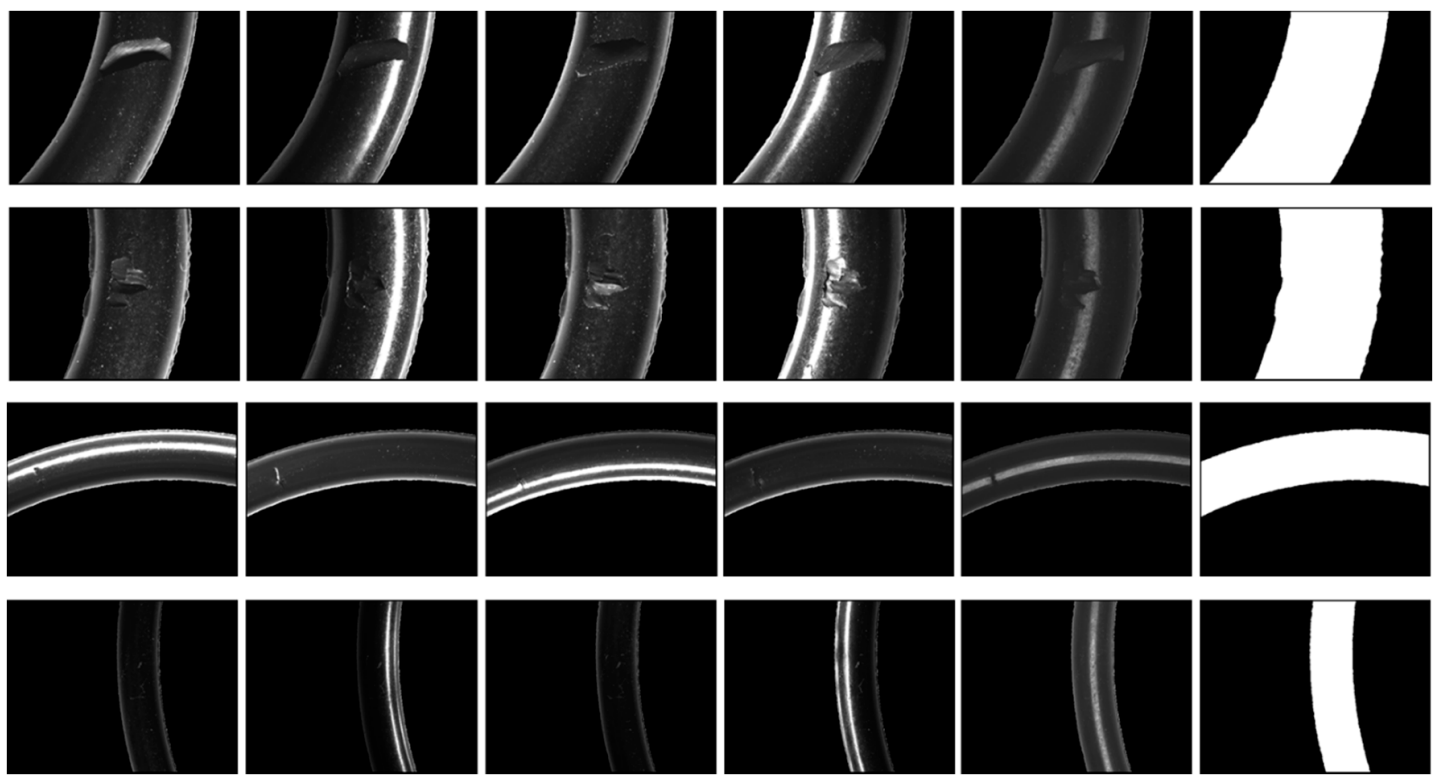

Figure 18. The schematic diagram of DPS samples. Each row is a set of sample capture with six light sources. The last row is a sample of non-defect.
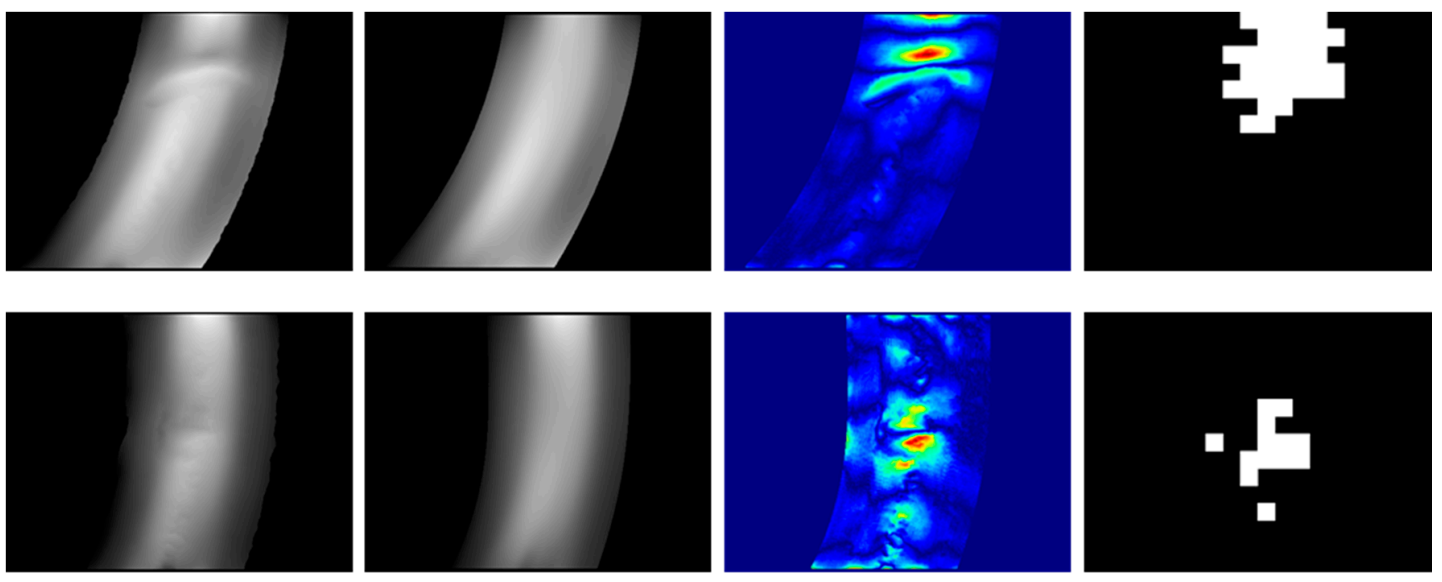

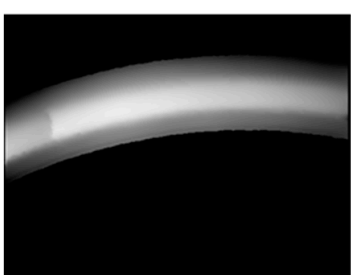

(a)

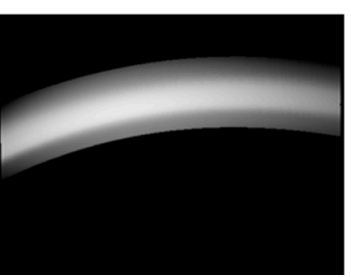

(b)

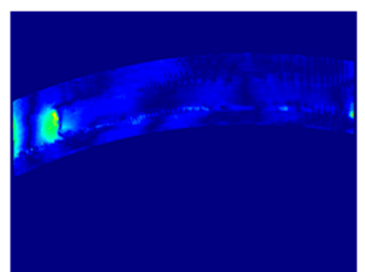

(c)

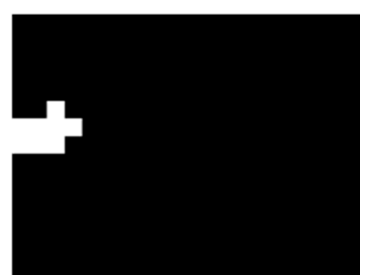

(d)

Figure 19. Defects detection processing with photometric stereo model $M_{\mathrm{P}}$. (a) Photometric stereo model $M_{\mathrm{P}}$ (Gray scale), those models reconstructed by corresponding images in Figure 18; (b) Smooth surface model $M_{\mathrm{S}}$ (Gray scale), this model is the smooth version of Photometric stereo model $M_{\mathrm{P}}$, create by the method in this paper Section 2.2; (c) the deviation model $M_{\mathrm{D}}$, this model created by subtracting $(\mathbf{a}, \mathbf{b})$ (jet color map and mapped into range 0 500 $\mu \mathrm{m}$ ); (d) Candidate area (Binary images), which is produced by sliding windows with (c). 
The reconstruction time consuming is about $30 \mathrm{~s}$ and the defect detection time consuming is about $30 \mathrm{~s}$.

\subsubsection{DK (Database of Raw Data with Profilometer)}

There were 160 defects and 160 non-defects included. The defect samples included 60 samples with flow marks and 100 samples with non-fills and indentations. Each sample was reconstructed using optical profilometer and the reconstruction raw data were acquired. Detailed specification of K optical profilometer is shown in Table 2.

Table 2. Specification of optical profilometer VR-3100.

\begin{tabular}{cc}
\hline Name & Specification and Parameter \\
\hline Trademark and model & VR-3100 \\
Magnification & $12 \times$ \\
FOV(field of view) & $24 \times 18 \mathrm{~mm}^{2}$ \\
Resolution & $1024 \times 768$ \\
Working distance & $75 \mathrm{~mm}$ \\
Pixel size & $23.4375 \times 23.4375 \mathrm{~m}^{2}$ \\
Repeatability (Vertical) & $0.5 \mu \mathrm{m}$ \\
Repeatability (horizontal) & $1 \mu \mathrm{m}$ \\
\hline
\end{tabular}

In this experiment, we establish the database with reconstruction raw data in $2.5 \mathrm{D}$ format and binary the 2.5D model to get binary image before defect detection. Then, we execute the defect detection we proposed (Figure 5 yellow region). We set the defect depth was $0.08 \mathrm{~mm}$ to detect surface defect, which depth is based on ISO standard. The other defect detection condition is same as in the DPS database. The reconstruction time consuming is about $1 \mathrm{~min}$. The defect detection time consuming is about $30 \mathrm{~s}$ (Figure 20).
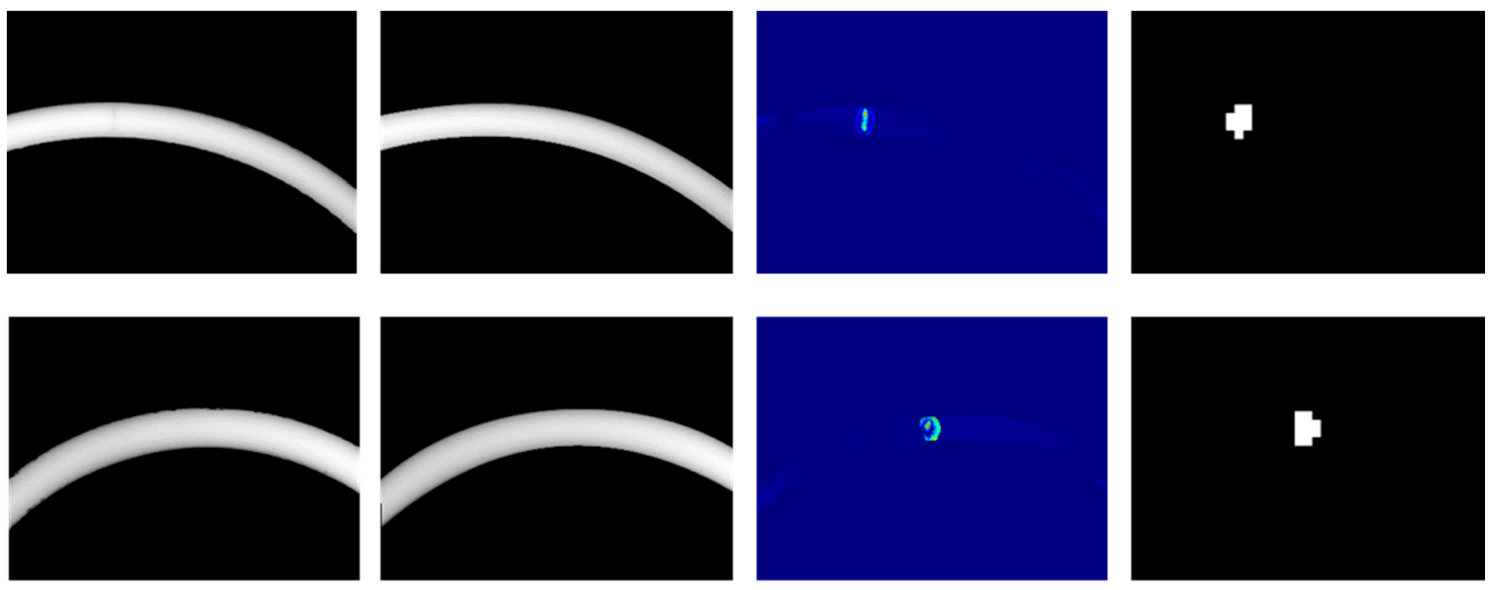

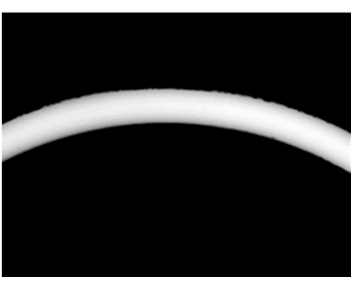

(a)

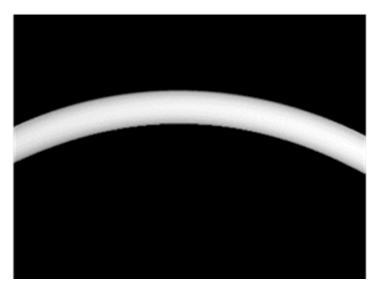

(b)

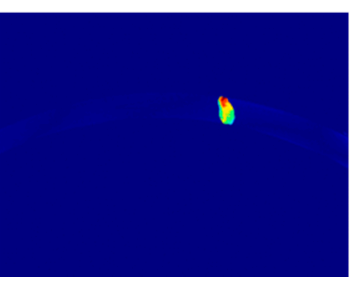

(c)

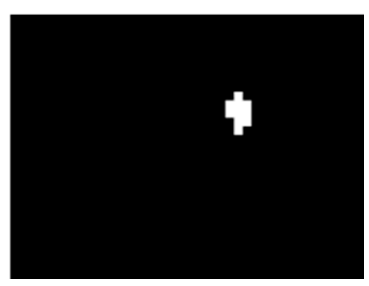

(d)

Figure 20. Defect detection processing with optical profilometer. (a) Model of optical profilometer reconstructed (Gray scale); (b) Smooth surface model $M_{S}$ (Gray scale), this model is the smooth version of (a), create by the method in this paper Section 2.2; (c) The deviation model $M_{\mathrm{D}}$, this model created by subtracting $(\mathbf{a}, \mathbf{b})$ (jet color map and mapped into range $0 \sim 500 \mu \mathrm{m})$; (d) Candidate area (Binary images), which is produced by sliding windows with (c). 


\subsection{Database for Dimension Measurement}

\subsubsection{DVT (Database of the Verified Samples Be Made of T6061)}

The standard piece was processed and manufactured with lathe using aluminum alloy (T6061). The products are shown in Figure 13. The standard piece was manufactured for 5 dimensions and 5 pieces for each dimension. Therefore, we had 25 pieces. The dimensions for the manufacturing process were as follows: internal dimension $d_{1}=(5,10$, $15,20,25) \mathrm{mm}$ and cross-sectional diameter $d_{2}=1.78 \mathrm{~mm}$.

To calculate the MAE (Mean Absolute Error) for each O-Ring, we classified the metal $\mathrm{O}$-Ring of each dimension according to the measurement results. The schematic diagram of the metal O-Ring is shown in Figure 21.
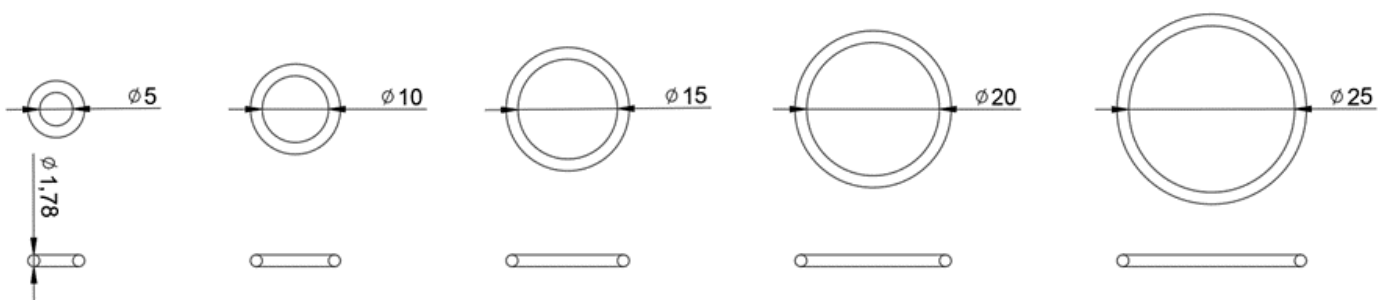

(a)

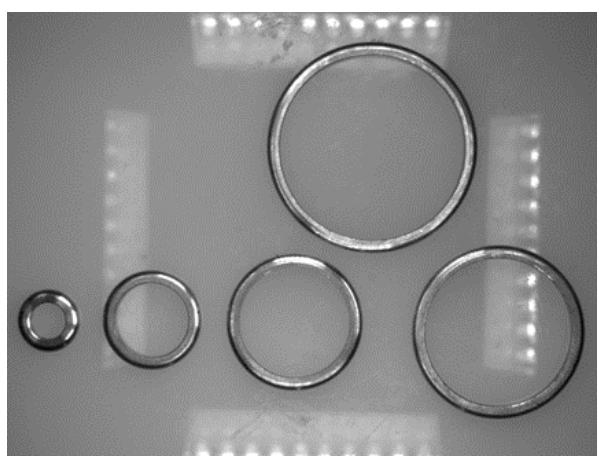

(b)

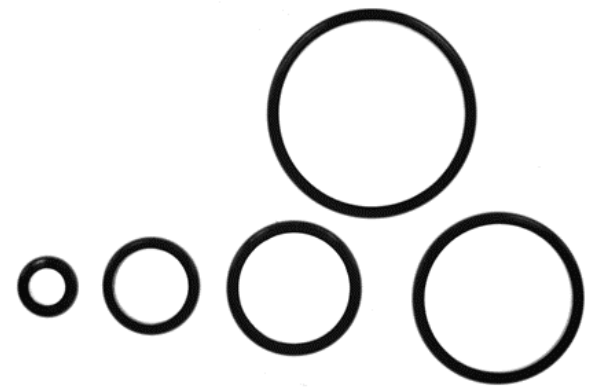

(c)

Figure 21. (a) CAD of the metal O-Rings; (b) metal O-Ring image (top light source); (c) metal O-Ring image (backlight source).

We put all of the metal O-Rings above the button light source in the dimension measurement mechanism (Figure $3 b$ ). Then, trigger the camera and light source at the same time for each sample to capture images and establish this database. Besides, we used the same measurement parameter to measure each size sample.

\subsubsection{DSI (Database of the Standard Sample Regulated by ISO}

This database collected a set of samples with dimensions that conformed to ISO 36012:2016 [19], ISO16031-1:2002 [20], and ISO16031-2:2003 [21]. All samples were samples defined with the size identification code of the above three specifications. The inside diameters $d_{1}$, cross-sections $d_{2}$, and O-Ring tolerances of the size identification code are shown in Table 3.

To calculate the MAE of each size O-Ring, and we put each O-Ring on the measurement platform 30 times repeatedly. This action can provide some deformation. We can measure the algorithm stability by this action. The action schematic diagram is shown in Figure 22. Besides, we used the same measurement parameter to measure each size sample. 

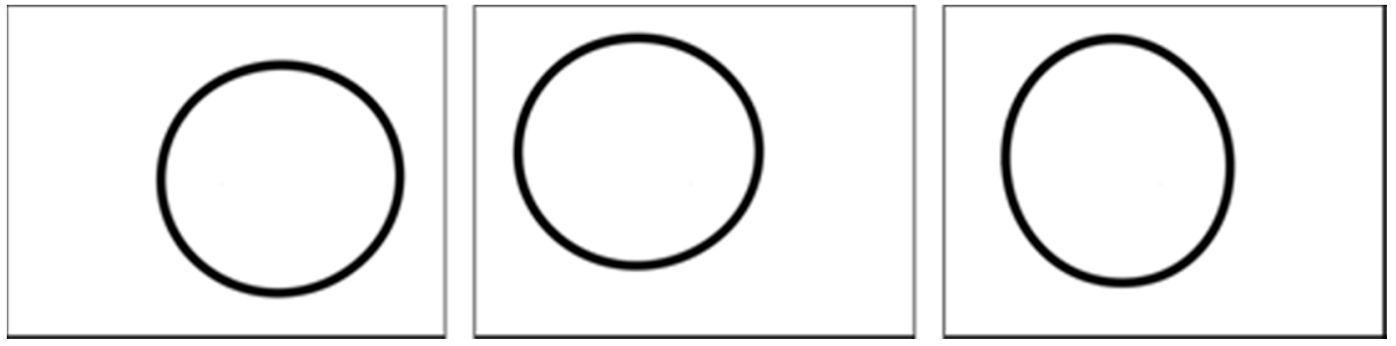

Figure 22. Schematic diagram of the O-Ring repeatedly on the object plane.

Table 3. Inside diameters, cross-sections, and tolerances for O-Rings.

\begin{tabular}{ccccc}
\hline \multirow{2}{*}{ SIC (Size Identification Code) } & \multicolumn{2}{c}{ Internal Diameter $\boldsymbol{d}_{\mathbf{1}} \mathbf{( m m )}$} & \multicolumn{2}{c}{ Cross-Section $\boldsymbol{d}_{\mathbf{2}}(\mathbf{m m})$} \\
\cline { 2 - 5 } & Min. & Max. & Min. & Max. \\
\hline-005 & 2.44 & 2.69 & 1.70 & 1.85 \\
-006 & 2.77 & 3.02 & 1.70 & 1.85 \\
-007 & 3.56 & 3.81 & 1.70 & 1.85 \\
-008 & 4.34 & 4.60 & 1.70 & 1.85 \\
-009 & 5.16 & 5.41 & 1.70 & 1.85 \\
-010 & 5.94 & 6.20 & 1.70 & 1.85 \\
-011 & 7.52 & 7.77 & 1.70 & 1.85 \\
-012 & 9.12 & 9.37 & 1.70 & 1.85 \\
-013 & 10.69 & 10.95 & 1.70 & 1.80 \\
-014 & 12.29 & 12.55 & 1.70 & 1.85 \\
-015 & 13.87 & 15.72 & 1.70 & 1.85 \\
-016 & 15.47 & 17.30 & 1.70 & 1.85 \\
-017 & 17.04 & 18.90 & 1.70 & 1.85 \\
-019 & 18.64 & 20.50 & 1.70 & 1.85 \\
-020 & 20.19 & 22.10 & 1.70 & 1.85 \\
-022 & 21.79 & 23.67 & 1.70 & 1.85 \\
\end{tabular}

3.2.3. DSP (Database of the Standard Sample with External Pressure)

This database included O-Rings with size identification codes from -017 to -020 , regulated by the ISO [19-21]. We pressured those O-Rings until deformation and fixed them on the object platform. Afterward, we captured all of those O-Ring to establish this database (Table 4). All of measurement conditions and parameter is same with DSI database.

Table 4. Table of DPS (database of photometric stereo model) with SIC (size identification code) and image index.

SIC $\quad$ Image Index


Table 4. Cont.

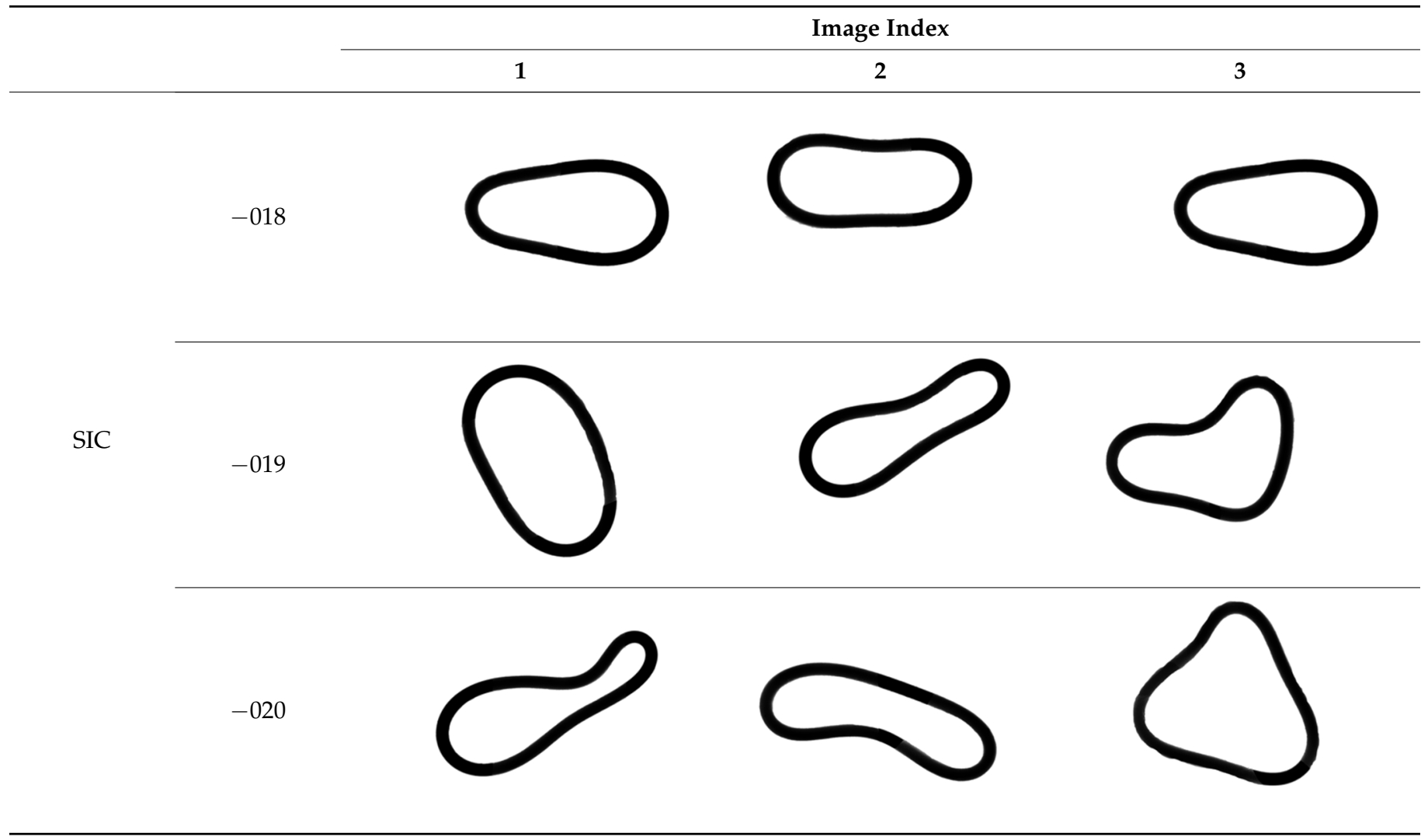

\section{Experiment Results and Discussion}

\subsection{Defection Detection}

The DPS result employed in the defect detection process is shown in Table 5 and Figure 23. In Figure 23, We successfully detect a tiny defect. The depth of this defect is $90 \mu \mathrm{m}$ and in this experiment, we did not have any defects to miss detection. However, there is little amount of false alarm. Because the photometric stereo method in this paper was based on regression, which type of method cannot provide accurate relative height. There are some tiny scratched at the O-Rings surface indeed, but not deep enough to be defective. Those type of sample is shown in Figure 24.

Table 5. Confusion matrix of the defect result with DPS.

\begin{tabular}{cccc}
\hline & & \multicolumn{2}{c}{ True Condition } \\
\cline { 3 - 4 } & & Positive & Negative \\
\hline \multirow{2}{*}{ Predicted Outcome } & Positive & 160 & 11 \\
& Negative & 0 & 149 \\
\hline Accuracy & \multicolumn{2}{c}{$96.56 \%$} \\
\hline Precision & \multicolumn{2}{c}{$93.57 \%$} \\
Recall & & $100 \%$ \\
\hline
\end{tabular}

To prove our suppose, which the reason for overkill from regression type photometric stereo. We use optical profilometer to establish the DK database. Then, we set a fixed threshold was $80 \mu \mathrm{m}$. In this experiment, we get a pretty good result. The DK result in the defect detection process is shown in Table 6 and Figure 25. This result can prove the processing we proposed was general. To improve reconstruction accuracy can improve the outcome of defect detection. On the other hand, we detect the same tiny defect O-Ring in optical profilometer reconstructed result. It also can successfully detect small defects. 


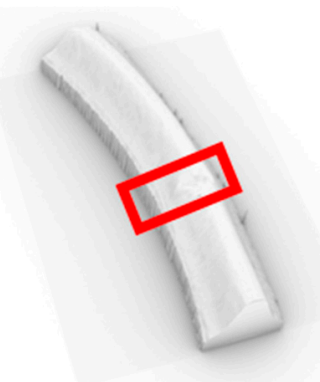

(a)

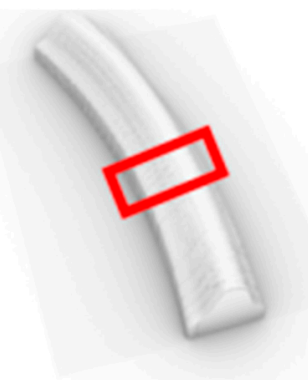

(b)

(c)

Figure 23. A tiny defect on the O-Ring's surface (a) Model of photometric stereo produce $M_{\mathrm{P}}$; (b) smooth model $M_{S}$; (c) subtracting result on defect candidate area.

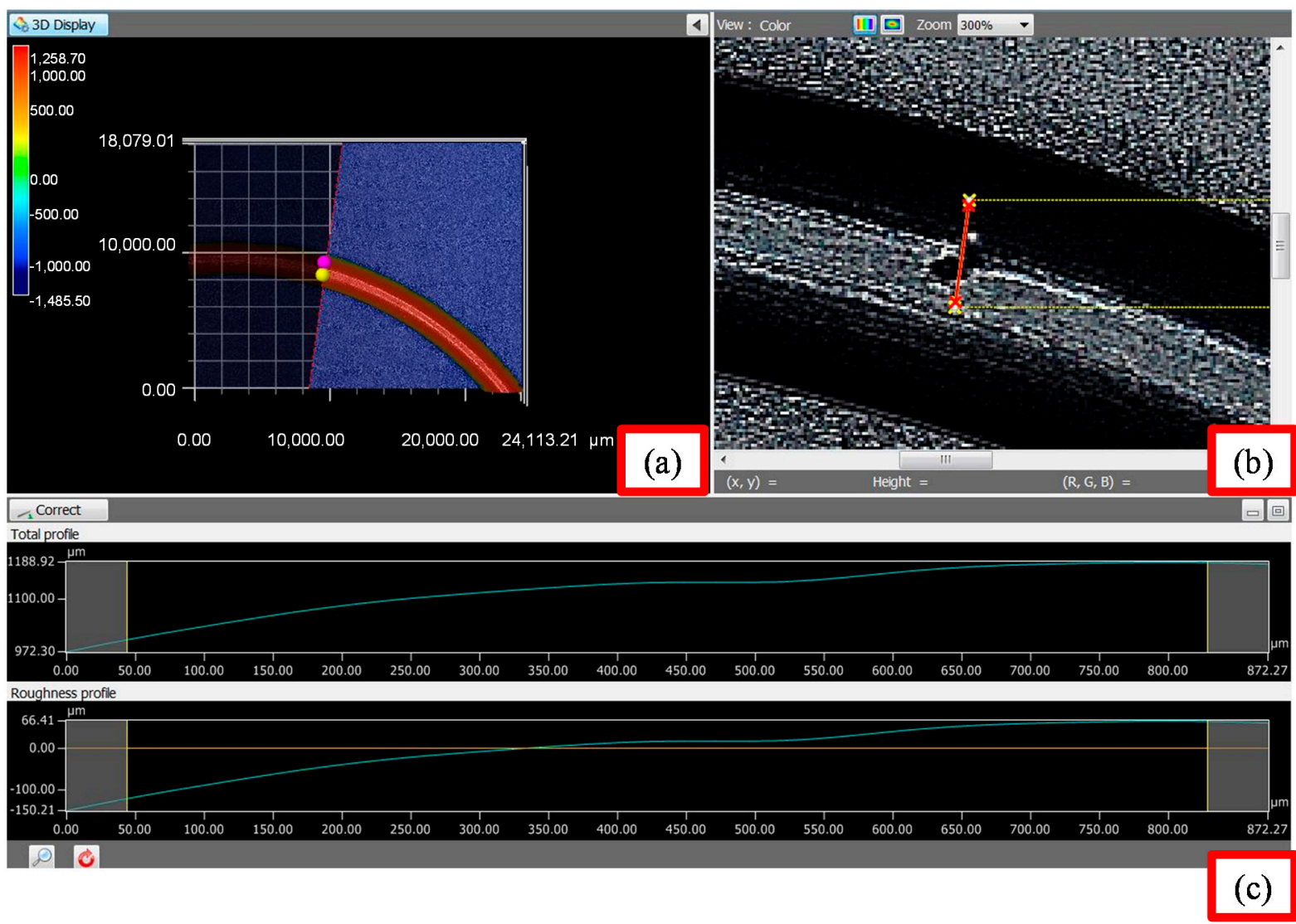

Figure 24. O-Ring sample with the tiny scratched measured by optical profilometer. (a) 3D model of the tiny scratched; (b) 2D image of the tiny scratched; (c) Measurement result of the tiny scratched. 
Table 6. Confusion matrix of the defect result with DK.

\begin{tabular}{cccc}
\hline & & \multicolumn{2}{c}{ True Condition } \\
\cline { 3 - 4 } & & Positive & Negative \\
\hline \multirow{2}{*}{ Predicted Outcome } & Positive & 160 & 0 \\
\cline { 2 - 4 } & Negative & 0 & 160 \\
\hline Accuracy & & $100 \%$ \\
\hline Precision & & $100 \%$ \\
\hline Recall & & $100 \%$ \\
\hline
\end{tabular}

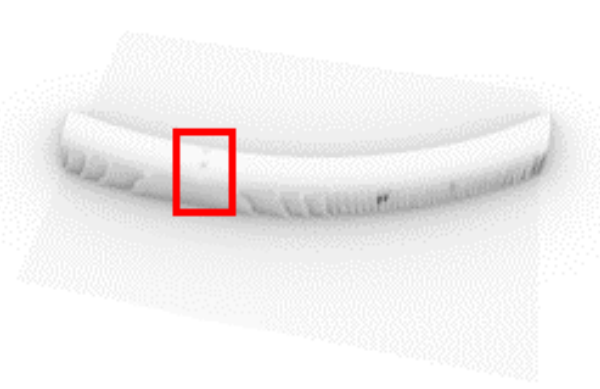

(a)

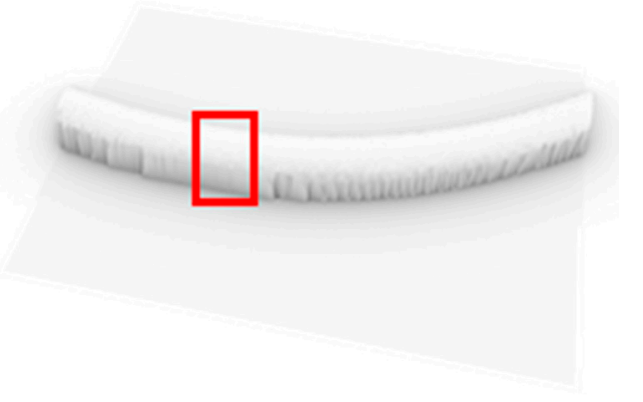

(b)

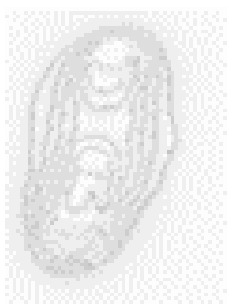

(c)

Figure 25. (a) optical profilometer model; (b) smooth model $M_{\mathrm{S}}$; (c) subtracting result on defect candidate area.

\subsection{Dimension Measurement}

We compared the Ramer-Douglas-Peucker algorithm result points $A$ and contour $C$ with the method $B_{\mathrm{p}}$ we proposed, all of those points through homography matrix and Euclidean distance to calculate distance. Then calculated the internal and cross-section diameter. On the other hand, we employed the mean absolute error to estimate loss $L$ to evaluate measurement results and stability. The formula of the loss $L$ is shown in Equation (21).

$$
L=\Sigma\left(\left|v-d_{\mathrm{S}}\right|\right) / n
$$

The $d_{\mathrm{s}}$ was the median size between the maximum allowable dimension and minimum allowable dimension from ISO. The loss $L$ was acquired using the median size $d_{\mathrm{s}}$, which deducted the measurement result $v$, and the $n$ is the number of the same size O-Ring samples.

The DVT measurement results of internal and cross-section diameters are shown in Figure 26a,b. Moreover, the DSI measurement results of internal and cross-section diameters are shown in Figure 26c,d. In addition, the DSP measurement results for the internal and cross-section diameters are shown in Figure 26e,f. All of the results display our processing can improve measurement result. 


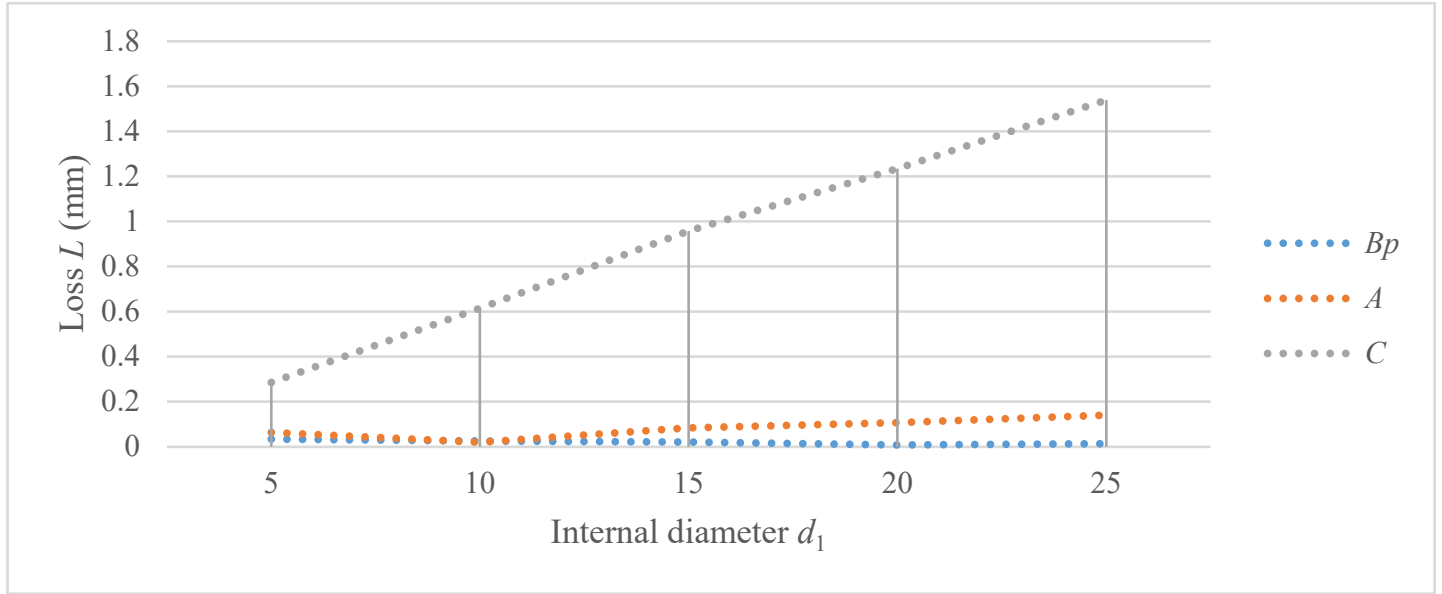

(a)

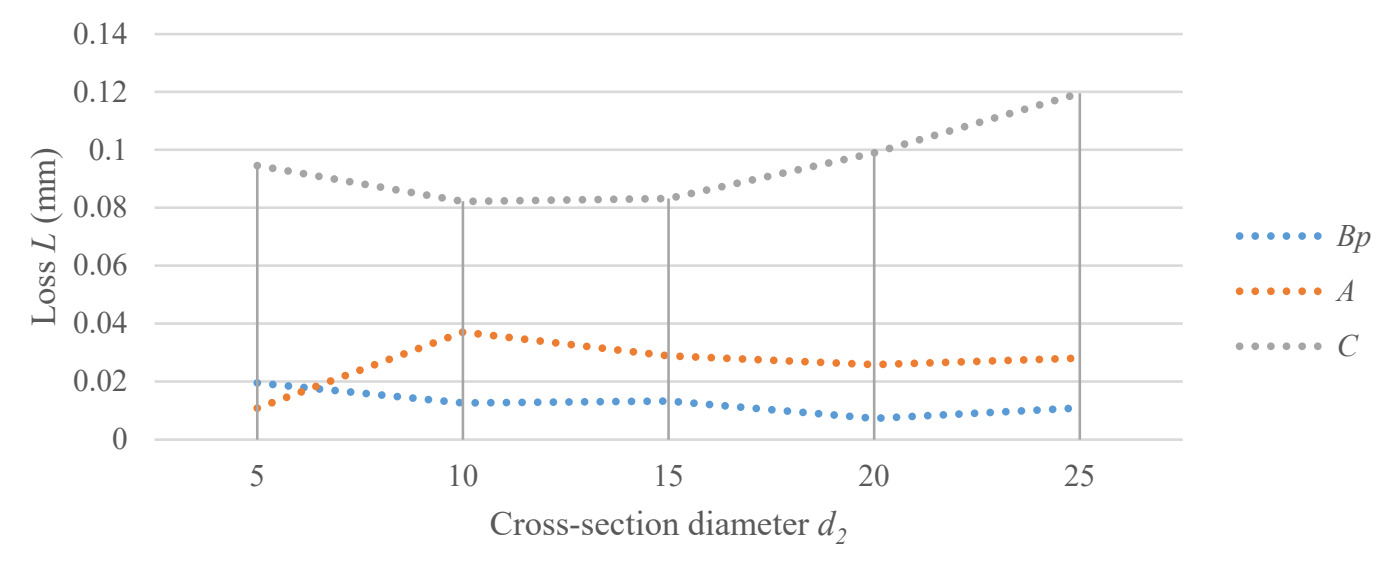

(b)

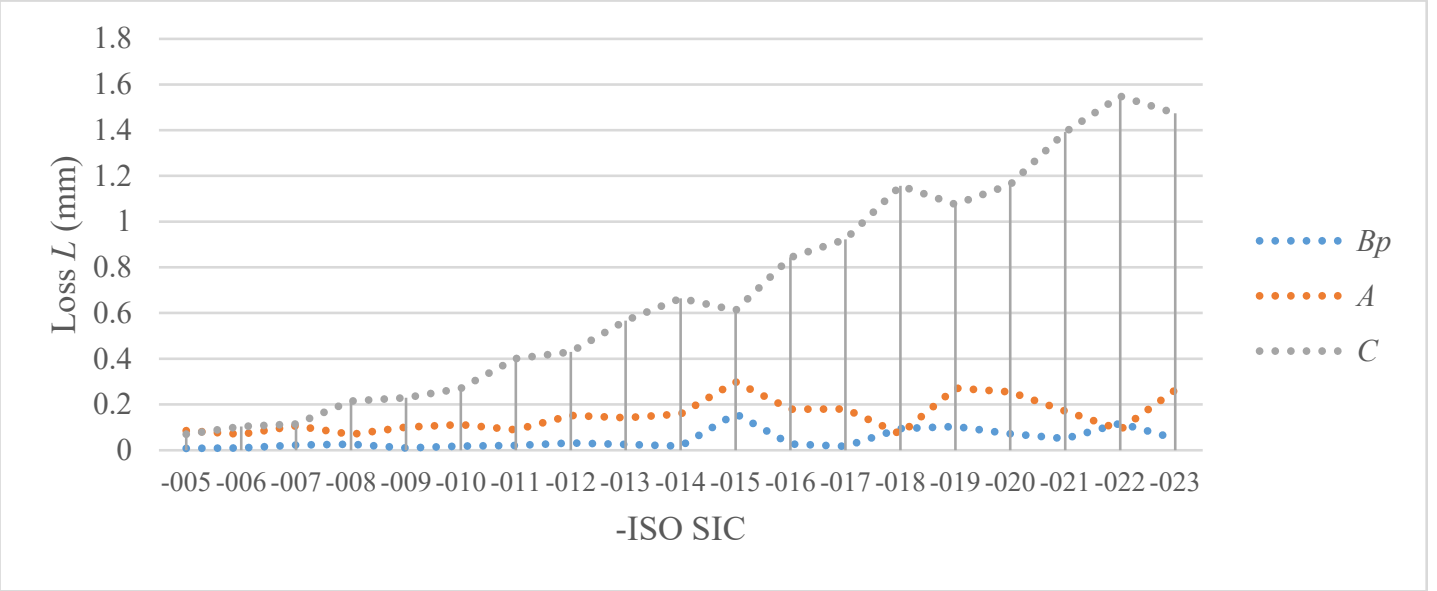

(c)

Figure 26. Cont. 


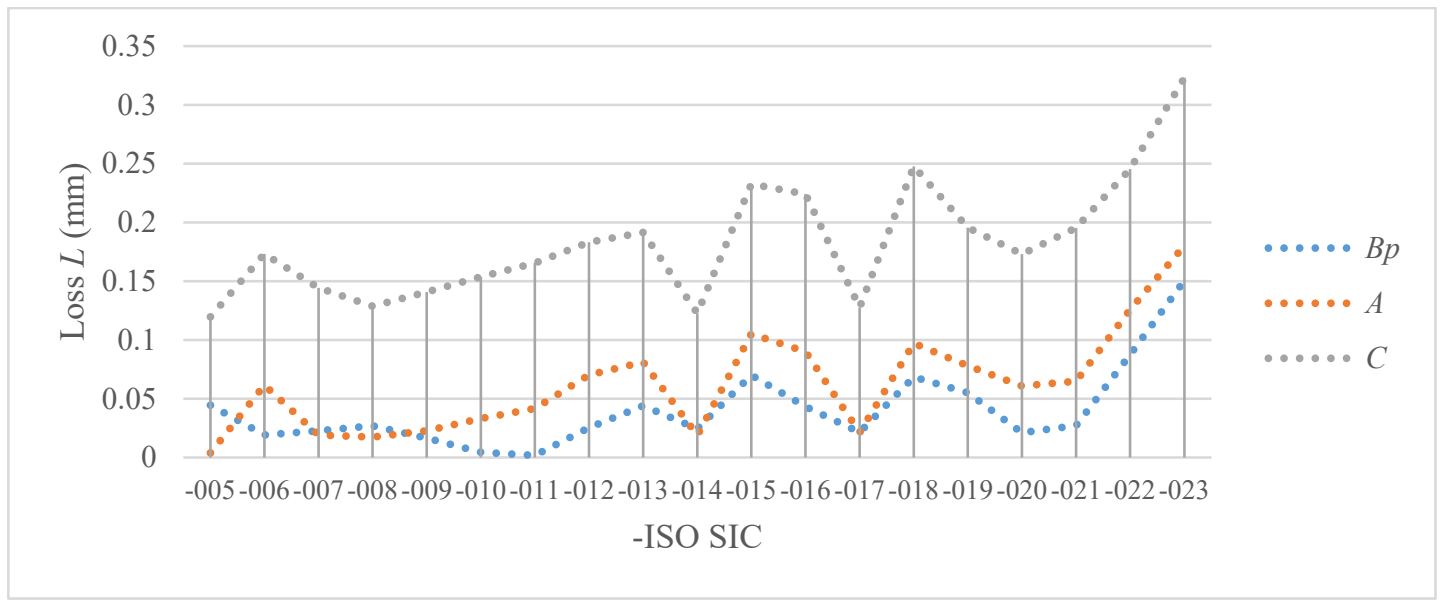

(d)

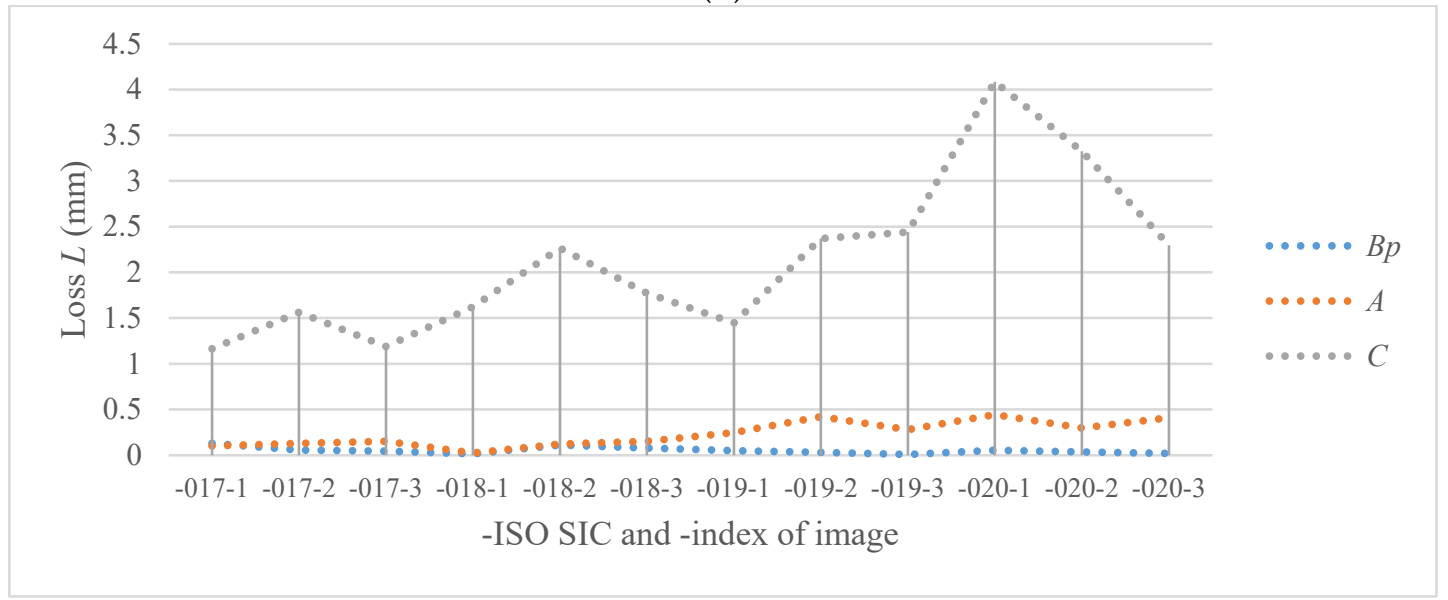

(e)

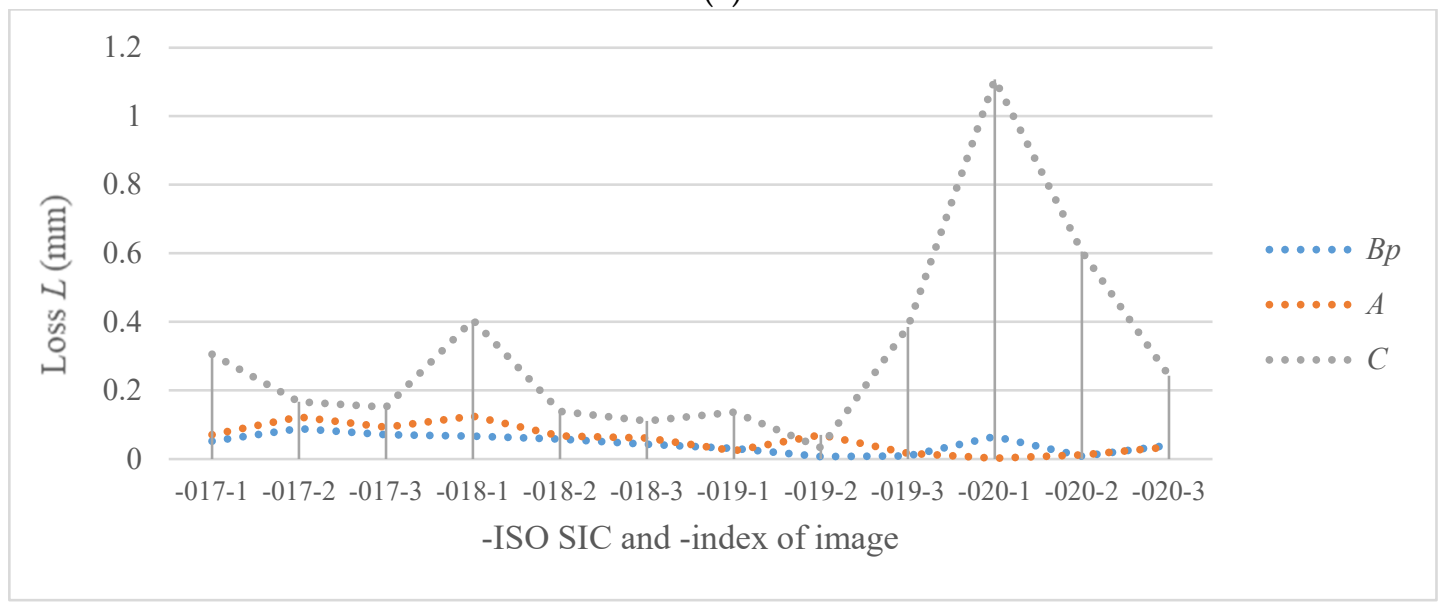

(f)

Figure 26. (a) Result of $d_{1}$ measurement with DVT; (b) result of $d_{2}$ measurement with DVT; (c) result of $d_{1}$ measurement with DSI; (d) result of $d_{2}$ measurement with DSI; (e) result of $d_{1}$ measurement with DSP; (f) result of $d_{2}$ measurement with DSP.

In those results, we can prove that the method we proposed can further improve the measurement error from edge protrusions. The detailed comparison is shown in Figure 27. There is some small noise at the O-Ring edge (Figure 27a). To improve the measurement, we ignore those protrusions using Ramer-Douglas-Peucker algorithm (Figure 27b). However, this method could lose the real edge of O-Ring too much from very large threshold. Such as the blue line and green line in Figure 27b, both lines were the measurement result 
using Ramer-Douglas-Peucker algorithm. Moreover, if we set the threshold too small, the algorithm loses the ability to ignore edge protrusions.

To further reduce measurement error, we proposed a new edge extracting algorithm in Section 2. This algorithm using a large threshold in Ramer-Douglas-Peucker algorithm. We fitted each contour $C$ between every two points $A$ by using the cubic Bézier curve. The cubic Bézier curve is shown in Figure 27c. In Figure 27c, red points are Ramer-DouglasPeucker algorithm result points and cubic Bézier control points $\mathrm{p}_{00}$ and $\mathrm{p}_{03}$, and green points are cubic Bézier control points $\mathrm{p}_{01}$ and p02 by Equation (19) and the yellow curve was the fitting result of our cubic Bézier fitting algorithm.

Thus, we can ignore edge protrusions and guarantee real edge information be keeping. Then, we use homography to calculate dimension.

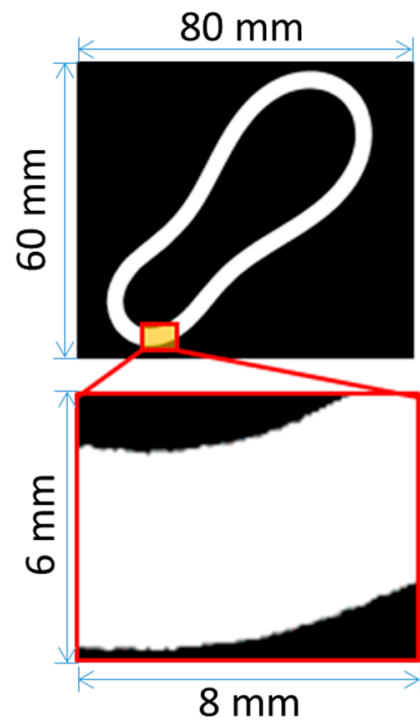

(a)

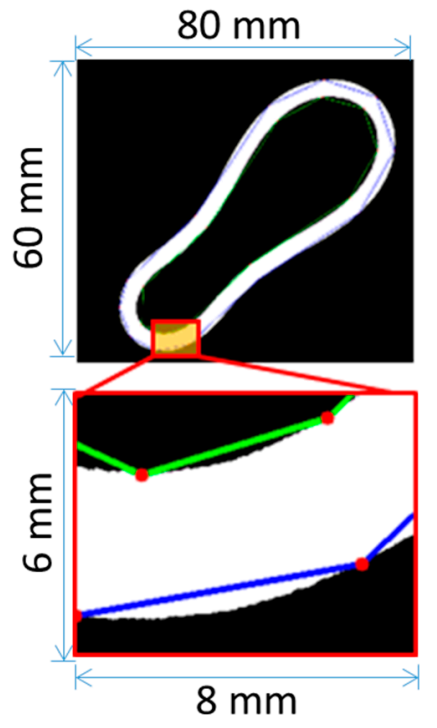

(b)

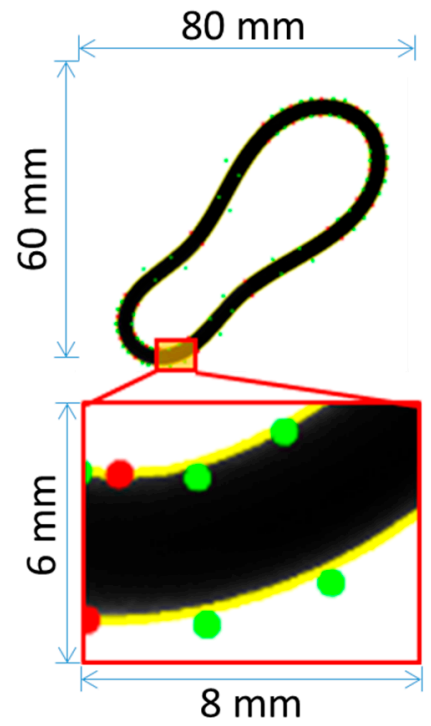

(c)

Figure 27. (a) Schematic diagram of the edge of contour with noise and protrusions; (b) Schematic diagram of approximate points with loss information by Ramer-Douglas-Peucker algorithm in large threshold. The red points are the algorithm result points, and the green and blue lines represent internal edge-tracking result and external edge tracking result; (c) Schematic diagram of fitting results found using cubic Bézier curves. which green points are cubic Bézier control points $\mathrm{p}_{01}$ and $\mathrm{p}_{02}$ by Equation (19). The yellow curve was the fitting result of our cubic Bézier fitting algorithm.

To verify measurement stability, we used the STD (standard deviation) as an index for measurement stability. The measurement results of $d_{1}$ and $d_{2}$ are shown in Tables 7 and 8 . This result proves the measurement processing we proposed is the most stable in those three algorithms. On the other hand, most of the time consumed in measurement processing was homography projective and Euclidean distance calculation. Our algorithm's result points were extracting by de Casteljau's algorithm. In de Casteljau's algorithm, we can reduce extracted resolution to reduce the total measurement time. So the time consuming is less than the contour method.

Table 7. Table of the internal diameter measurement result via STD.

\begin{tabular}{cccc}
\hline Unit: $\mathbf{m m}$ & \multicolumn{3}{c}{ Methods of Measurement } \\
\hline Name of Database & $\boldsymbol{B}_{\mathbf{p}}$ & $\boldsymbol{A}$ & $\boldsymbol{C}$ \\
\hline DVT & 0.01401 & 0.04627 & 0.47170 \\
\hline DSI & 0.04606 & 0.07764 & 0.48437 \\
\hline DSP & 0.03703 & 0.13972 & 0.87662 \\
\hline
\end{tabular}


Table 8. Table of cross-section diameter measurement result via STD.

\begin{tabular}{cccc}
\hline Unit: $\mathbf{m m}$ & \multicolumn{3}{c}{ Methods of Measurement } \\
\hline Name of Database & $\boldsymbol{B}_{\mathbf{p}}$ & $\boldsymbol{A}$ & $\boldsymbol{C}$ \\
\hline DVT & 0.00741 & 0.01657 & 0.03544 \\
\hline DSI & 0.03424 & 0.04591 & 0.05424 \\
\hline DSP & 0.02697 & 0.04145 & 0.29591 \\
\hline
\end{tabular}

\section{Conclusions}

We developed an affordable AOI system for O-Ring inspection and dimension measurement. The total cost of the system we proposed is only $\$ 1500$ USD. The system not only achieved a $100 \%$ recall rate, but the accuracy reached $95.56 \%$ in the collected dataset. We also detected some defects were not yet regulated by the ISO [9]. Meanwhile, we effectively avoided the false negative state caused by surface dirt. To detect of each O-Ring only take $30 \mathrm{~s}$ because the detect algorithm is based on 2-D data format. On the other hand, the smooth model produced by the detect algorithm can be establishing a deep learning database. We also successfully detected the oxidized defection, which ISO didn't mention. The rubber oxidized model is shown in Figure 28.

Alternatively, we proposed a new measurement method. The algorithm we proposed was experimentally verified to be more accurate, more stable, and faster. The algorithm method improves the Ramer-Douglas-Peucker algorithm real edge lose problem and keep the real edge information at the same time. Moreover, this algorithm time consuming can be reduced using de Casteljau's algorithm-extracting resolution.

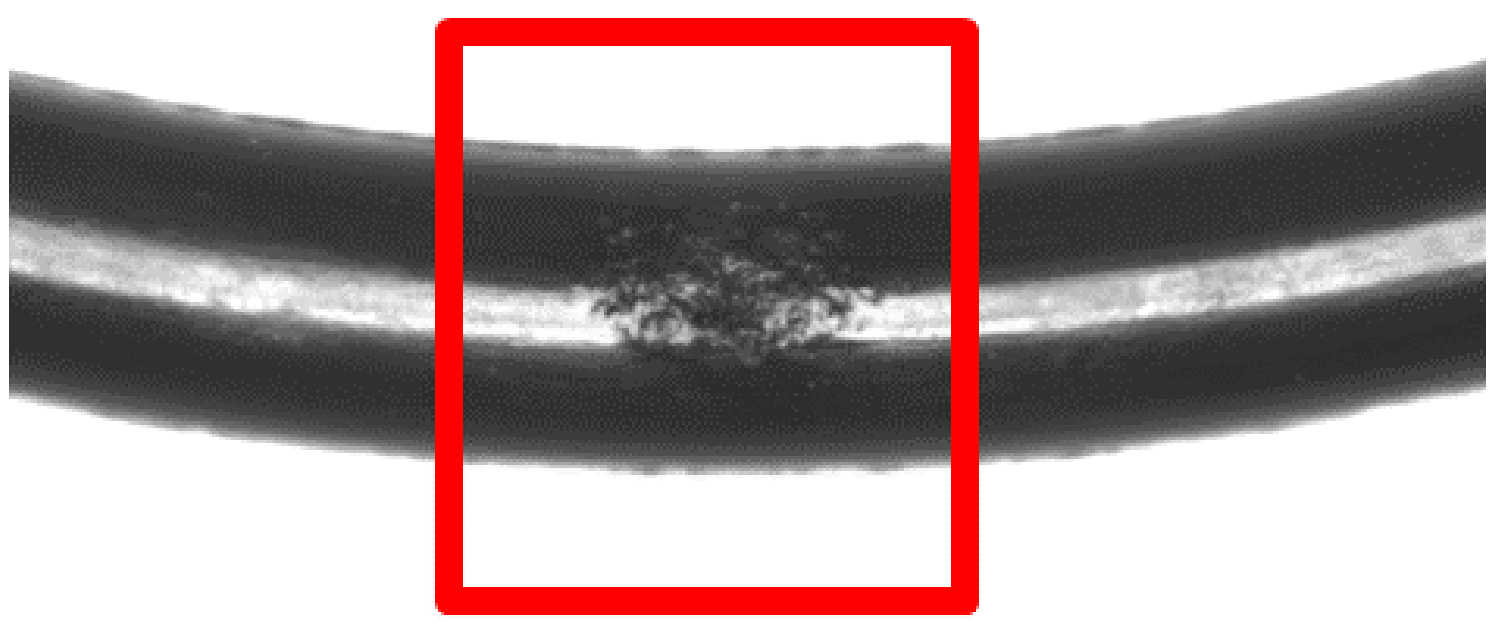

(a)

Figure 28. Cont. 


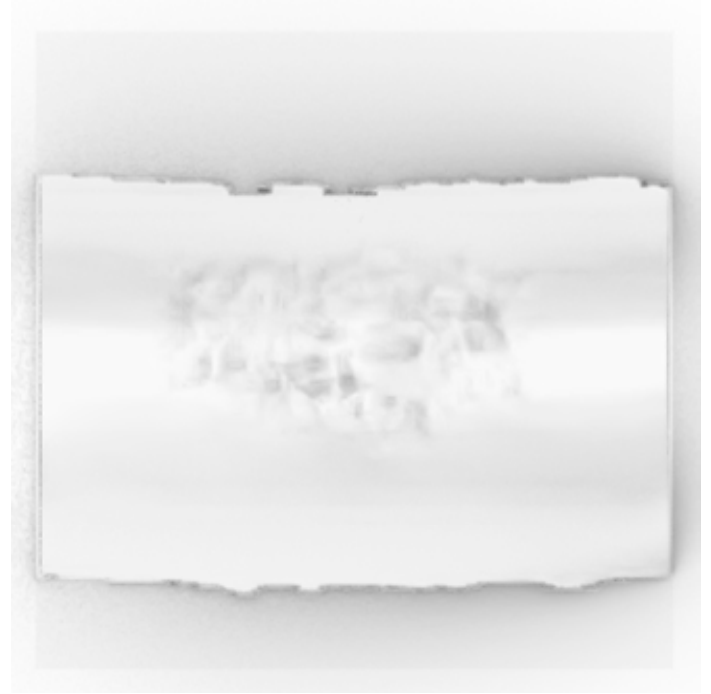

(b)

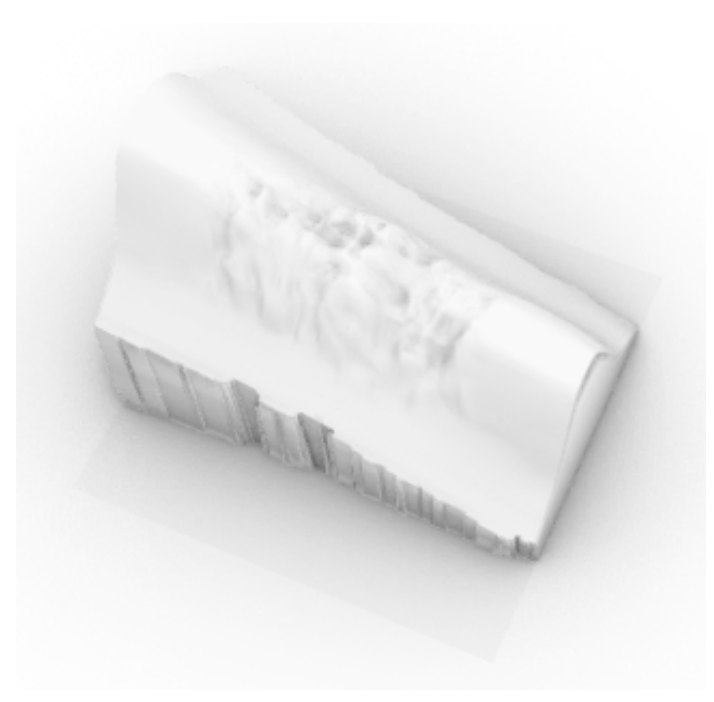

(c)

Figure 28. (a) Image of the rubber oxidized model; (b) rubber oxidized model (top view); (c) rubber oxidized model (perspective view).

Author Contributions: Conceptualization, C.-C.H. and F.-S.Y.; methodology, C.-C.H. and F.-S.Y.; software, F.-S.Y.; validation, C.-C.H. and F.-S.Y.; formal analysis, C.-C.H. and L.-C.C.; investigation, C.-C.H. and L.-C.C.; resources, C.-C.H.; data curation, F.-S.Y.; writing-original draft preparation, F.-S.Y.; writing-review and editing, C.-C.H.; visualization, C.-C.H.; supervision, C.-C.H.; project administration, C.-C.H.; funding acquisition, C.-C.H. All authors have read and agreed to the published version of the manuscript.

Funding: This article is for the Ministry of Science and Technology, Taiwan, grant number 107-2628E-027-002-MY3 and 109-2622-E-027-016-CC3, thanks to the support of the Ministry of Science and Technology, Taiwan.

Institutional Review Board Statement: Not applicable.

Informed Consent Statement: Not applicable.

Conflicts of Interest: This research received no external funding.

\section{References}

1. Peng, G.; Zhang, Z.; Li, W. Computer vision algorithm for measurement and inspection of O-Rings. Measurement 2016, 94, 828-836. [CrossRef]

2. Ho, C.-C.; Lu, J.-J.; Li, P.-C. Development of auto defect inspection system for cell phone silicone rubber gasket. In Proceedings of the 10th International Symposium on Precision Engineering Measurements and Instrumentation (ISPEMI 2018), Kunming, China, 8-10 August 2018.

3. Husmann, T.; Kreimeier, D. Measurement of radial-axial rolled rings by using image processing and thermography. In Key Engineering Materials; Trans Tech Publ.: Stafa, Switzerland, 2015; pp. 278-283.

4. Husmann, S.; Husmann, T.; Meier, H. Measurement of rings via image processing during and after radial-axial ring rolling. In Applied Mechanics and Materials; Trans Tech Publ.: Stafa, Switzerland, 2015; pp. 136-143.

5. Dokken, T.; Dæhlen, M.; Lyche, T.; Mørken, K. Good approximation of circles by curvature-continuous Bézier curves. Comput. Aided Geom. Des. 1990, 7, 33-41. [CrossRef]

6. Lin, J.-F. Use of Cubic Bézier Curve Approximation of Fourth-Order Bézier Curve. Master's Thesis, National Chung Cheng University, Chiayi, Taiwan, 2012.

7. Bézier, P. Courbes et surfaces pour la CFAO. Tech. l'Ingénieur 1992, A1440, 1-18.

8. Goldapp, M. Approximation of circular arcs by cubic polynomials. Comput. Aided Geom. Des. 1991, 8, 227-238. [CrossRef]

9. Urbikain, G.; Alvarez, A.; López de Lacalle, L.N.; Arsuaga, M.; Alonso, M.A.; Veiga, F. A reliable turning process by the early use of a deep simulation model at several manufacturing stages. Machines 2017, 5, 15. [CrossRef]

10. Urbikain, G.; Perez, J.M.; López de Lacalle, L.N.; Andueza, A. Combination of friction drilling and form tapping processes on dissimilar materials for making nutless joints. Proc. Inst. Mech. Eng. Part B J. Eng. Manuf. 2018, 232, 1007-1020. [CrossRef] 
11. Fluid Power Systems_O-Rings_Part 3: Quality Acceptance Criteria; ISO 3601-3:2005; International Organization for Standardization: Geneva, Switzerland, 2005.

12. Fluid Power Systems_-O-Rings_-Part 1: Inside Diameters, Cross-Sections, Tolerances and Designation Codes; ISO 3601-1:2012; International Organization for Standardization: Geneva, Switzerland, 2012.

13. Zhang, J. A New 3D Reconstruction Algorithm Based on Photometric Stereo. Master's Thesis, Northwestern Polytechnical University, Shaanxi, China, 2004.

14. Suzuki, S. Topological structural analysis of digitized binary images by border following. Comput. Vis. Graph. Image Process. 1985, 30, 32-46. [CrossRef]

15. Din, I.; Anwar, H.; Syed, I.; Zafar, H.; Hasan, L. Projector calibration for pattern projection systems. J. Appl. Res. Technol. 2014, 12, 80-86. [CrossRef]

16. Dubrofsky, E. Homography Estimation. Ph.D. Thesis, The University of British Columbia, Kelowna, BC, Canada, 2009.

17. Douglas, D.H.; Peucker, T.K. Algorithms for the reduction of the number of points required to represent a digitized line or its caricature. Cartogr. Int. J. Geogr. Inf. Geovisualization 1973, 10, 112-122. [CrossRef]

18. Boehm, W.; Müller, A. On de Casteljau's algorithm. Comput. Aided Geom. Des. 1999, 16, 587-605. [CrossRef]

19. Fluid Power Systems—O-Rings_Part 2: Housing Dimensions for General Applications; ISO 3601-2:2016; International Organization for Standardization: Geneva, Switzerland, 2016.

20. Aerospace Fluid Systems-O-Rings, Inch Series: Inside Diameters and Cross Sections, Tolerances and Size-Identification Codes-Part 1: Close Tolerances for Hydraulic Systems ISO; ISO 16031-1:2002; International Organization for Standardization: Geneva, Switzerland, 2002.

21. Aerospace Fluid Systems-O-Rings, Inch Series: Inside Diameters and Cross-Sections, Tolerances and Size-Identification Codes-Part 2: Standard Tolerances for Non-Hydraulic Systems; ISO 16031-2:2003; International Organization for Standardization: Geneva, Switzerland, 2003. 